\title{
Carriage of Neisseria Meningitidis in Low and Middle Income Countries of the Americas and Asia: A Review of the Literature
}

\author{
Lidia Serra $\cdot$ Jessica Presa $\cdot$ Hannah Christensen $\cdot$ Caroline Trotter
}

Received: December 26, 2019 / Published online: April 2, 2020

(C) The Author(s) 2020

\begin{abstract}
Introduction: Meningococcal colonization, or carriage, can progress to invasive meningococcal disease, a serious public health concern, with rapid progression of disease and severe consequences if left untreated. Information on meningococcal carriage and epidemiology in low/middle income American and Asian countries remains sparse. These data are crucial to ensure that appropriate preventive strategies such as vaccination can be implemented in
\end{abstract}

Digital Features To view digital features for this article go to https://doi.org/10.6084/m9.figshare. 11962566.

Electronic supplementary material The online version of this article (https://doi.org/10.1007/s40121020-00291-9) contains supplementary material, which is available to authorized users.

L. Serra $(\bowtie)$

Pfizer Vaccine Medical Development, Scientific and Clinical Affairs, Collegeville, PA, USA

e-mail: Lidia.Oliveira@pfizer.com

J. Presa

Pfizer Vaccines, Medical and Scientific Affairs,

Collegeville, PA, USA

H. Christensen

Bristol Medical School, Population Health Sciences, University of Bristol, Clifton, UK

C. Trotter

Department of Veterinary Medicine, University of Cambridge, Cambridge, UK these regions. The goal of this study was to summarize the Neisseria meningitidis carriage literature in low and middle income countries of the Americas and Asia.

Methods: Target countries were categorized as low and middle income according to the International Monetary Fund classification of low income/developing economies and middle income/emerging market economies, respectively. A PubMed search identified Englishlanguage publications that examined carriage in these countries. Studies reporting the epidemiology of N. meningitidis carriage or assessing risk factors for carriage were included.

Results: Fourteen studies from the Americas [Brazil $(n=7)$, Chile $(n=3)$, and Colombia, Cuba, Mexico, and Paraguay $(n=1$ each)] and nine from Asia [China $(n=2)$, India $(n=3)$, and Malaysia, Nepal, Philippines, and Thailand $(n=1$ each)] were identified; an additional Cuban study from the authors' files was also included. Studies were not identified in many target countries, and substantial diversity was observed among study methodologies, populations, and time periods, thereby limiting comparison between studies. The carriage rate in the Americas ranged from $1.6 \%$ to $9.9 \%$ and from $1.4 \%$ to $14.2 \%$ in Asia. Consistent risk factors for carriage were not identified.

Conclusions: There is a lack of comprehensive and contemporary information on meningococcal carriage in low and medium income countries of the Americas and Asia. Future 
carriage studies should incorporate larger representative populations, a wider age range, and additional countries to improve our understanding of meningococcal epidemiology and disease control.

Keywords: Asia; Carriage; Latin America; LMICs; Neisseria meningitidis

\section{Key Summary Points}

\section{Why carry out this study?}

Meningococcal colonization, or carriage, can progress to invasive meningococcal disease, a serious public health concern, with rapid progression of disease and severe consequences if left untreated.

Meningococcal carriage and epidemiology in low/middle income American and Asian countries is unclear, thus presenting a barrier to implementing effective vaccination strategies.

The goal of this study was to summarize the Neisseria meningitidis carriage literature in low and middle income countries of the Americas and Asia.

\section{What was learned from the study?}

Reported carriage prevalence was 1.6-9.9\% in the Americas and $1.4-14.2 \%$ in Asia.

Risk factors for carriage were inconsistent between studies.

Comprehensive regional carriage studies are needed to improve disease control.

\section{INTRODUCTION}

Invasive meningococcal disease (IMD) is caused by infection by the Gram-negative bacterium Neisseria meningitidis [1]. Globally, the incidence and mortality from meningococcal meningitis has been decreasing since 1990, although disparities persist regarding at-risk age groups and geographic distribution [2]. In 2017, more than 400,000 cases and nearly 30,000 deaths were attributed to meningococcal meningitis [3]. IMD has a characteristically rapid course; fatalities often occur within $24 \mathrm{~h}$ [1, 4]. Additionally, approximately one in five survivors of IMD have long-term and disabling sequelae [5].

Nearly all life-threatening IMD is attributable to six serogroups $(\mathrm{A}, \mathrm{B}, \mathrm{C}, \mathrm{W}, \mathrm{X}, \mathrm{Y})$ [1]. IMD incidence and the most prevalent disease-causing serogroups vary by age group, as well as geographically and temporally [6]. For instance, meningococcal serogroup A (MenA) has predominated in sub-Saharan Africa and Southeast Asia and meningococcal serogroup B (MenB) and serogroup C (MenC) have predominated in South America [6]. MenB is also the predominant disease-causing serogroup in several Western countries and regions $[7,8]$. During the past 5-10 years, considerable increases in IMD caused by meningococcal serogroup W (MenW) have been observed within several regions, including South America, Europe, Australia, and New Zealand [8, 9].

The ecological niche of $N$. meningitidis is the human nasopharynx [1]. Transmission of $N$. meningitidis through respiratory secretions does not always result in disease; this asymptomatic colonization of the mucosa of the upper respiratory tract is a phenomenon known as carriage. Acquisition of meningococci usually results from close contact with a carrier rather than directly from a patient with IMD, highlighting the importance of carriage in IMD epidemiology [10-12]. In high-income regions such as the United Kingdom, carriage acquisition is highest in adolescents and young adults [13]. In a recent longitudinal study of the acquisition of meningococcal carriage in sub-Saharan Africa, the highest carriage rates were in those aged 5-14 years [12]. Based on data from North America, Europe and Australasia, carriage is associated with behaviors common in adolescents and young adults, such as living in dormitories, patronizing bars, smoking, and sharing utensils or beverages [14-16]. Because most transmission occurs between carriers, using effective preventive strategies, such as vaccination, is important to decrease carriage rates in a population through both direct 
vaccination and herd effect, as has been illustrated by the use of meningococcal conjugate vaccines [17-19].

The lack of comprehensive data available for carriage, particularly in low and middle income countries outside of sub-Saharan Africa, limits elucidation of the epidemiology of IMD and carriage. These data are required to ensure that appropriate preventive strategies are implemented. The purpose of this review is to summarize the current literature regarding the carriage of $N$. meningitidis in low and middle income countries of the Americas and Asia. These data could inform healthcare authorities of regional disease burden and potentially influence existing meningococcal vaccination policies (summarized in Table S1).

\section{METHODS}

Low income/developing and middle income/ emerging market economies of the Americas and Asia were identified according to the International Monetary Fund classification (hereafter referred to as low and middle income countries, respectively) [20]. A PubMed search was conducted on November 11, 2019, of English-language publications from 1980 that examined carriage in low and middle income countries of the Americas (i.e., South America, Latin America, and the Caribbean) and Asia utilizing the search strings summarized in Table 1. Studies reporting the epidemiology of carriage, including overall, by serogroup, and by age group and/or assessing risk factors for carriage were included in the analysis. Any relevant articles from authors' files were also included. Narrative and systematic reviews, conference proceedings, and case studies were excluded and the reference lists of the identified studies were not systematically reviewed to identify other published studies. This article is based on previously conducted studies and does not contain any studies with human participants or animals performed by any of the authors.

Table 1 PubMed search strings

\begin{tabular}{lc}
\hline Region of Interest & PubMed search string \\
\hline The Americas (South America, Latin & (Neisseria meningitidis OR N meningitidis) AND (carriage OR colonization \\
America, and the Caribbean) & OR colonisation OR carrier) AND (Antigua OR Argentina OR Barbuda \\
& OR Bahamas OR Barbados OR Belize OR Bolivia OR Brazil OR Costa \\
& Rica OR Chile OR Colombia OR Dominica OR Dominican Republic OR \\
& Ecuador OR El Salvador OR French Guiana OR Grenada OR Grenadines \\
& OR Guatemala OR Guyana OR Guiana OR Haiti OR Honduras OR \\
& Jamaica OR Mexico OR Nevis OR Nicaragua OR Panama OR Paraguay \\
& OR Peru OR St Kitts OR St Lucia OR St Vincent OR Suriname OR \\
& Trinidad OR Tobago OR Uruguay OR Venezuela OR South America OR \\
& Latin America OR Caribbean) \\
& Neisseria meningitidis OR N meningitidis) AND (carriage OR colonization \\
& OR colonisation OR carrier) AND (Bangladesh OR Bhutan OR Brunei \\
& OR Brunei Darussalam OR Burma OR Cambodia OR China OR Fiji OR \\
India OR Indonesia OR Kiribati OR Laos OR Malaysia OR Maldives OR \\
Marshall Islands OR Micronesia OR Mongolia OR Myanmar OR Nauru \\
OR Nepal OR Palau OR Papua New Guinea OR Philippines OR Samoa \\
OR Solomon Islands OR Sri Lanka OR Thailand OR Timor-Leste OR \\
Tonga OR Tuvalu OR Vanuatu OR Vietnam) \\
\hline
\end{tabular}


For each identified publication, data regarding the population, setting, time frame, and epidemiology of carriage were extracted (Tables 2, 3). Additionally, the source and methodology to determine carriage were summarized (Tables S2, S3). As carriage should be considered in the context of disease epidemiology and vaccination strategies, a narrative review of these factors in the relevant regions and countries identified in this literature search has also been included.

\section{RESULTS}

\section{The Americas}

\section{Surveillance, Disease, and Vaccination}

The epidemiology of meningococcal disease and carriage in Latin America is not well characterized, which is largely attributed to disparities in surveillance activities between countries. Much of the data are obtained through a surveillance network system, which includes regional reference laboratories [19]. Although notification of IMD is compulsory in Latin America [21], the data are mainly passive, and only a few countries provide systematic and detailed reporting [22]. Thus, the quality of the reported data is often poor. Additionally, notable differences exist in healthcare systems, diagnostic methods, and the case definitions of IMD that may lead to great variability in epidemiologic data between countries [21].

The annual incidence of IMD in Latin America also varies widely between countries [21]. Currently, most cases of IMD in Latin America are sporadic and commonly caused by MenB and MenC, with the emergence of MenW in countries of the Southern Cone [21]. MenA disease is rarely observed in Latin America. Infants and young children in Latin America are particularly susceptible to sporadic cases of IMD, and adolescents and young adults are notably affected during outbreaks.

Meningococcal vaccines have not typically been included in national immunization programs in Latin America; rather, the use of meningococcal vaccination has often been in response to mass vaccination campaigns to control outbreaks and epidemics [23]. However, there are exceptions (Table S1) [24]. For instance, in 2010, Brazil introduced a MenC vaccine into their national immunization program in response to an increased incidence of IMD among infants and young children and recurring outbreaks throughout the country $[25,26]$. The vaccine was administered at age 3 and 5 months, with a booster dose given at age 12-15 months. Toddlers aged 12-23 months received one dose of the vaccine. No catch-up vaccination for older age groups was provided at that time [23]. Regional vaccination campaigns have also been conducted in Brazil. For example, the MenC vaccine was administered to individuals aged 10-24 years in the city of Salvador between May and August 2010 in response to an increased IMD burden in this age group in the first semester of the 2010 school year [27].

In addition, disease caused by MenW began to increase in Chile in 2010, accounting for 58\% of all cases by 2012 [28]. This was a hypervirulent strain often attributed to sequence type 11 clonal complex (cc11) [21], which caused an increase in mortality and long-term disabling sequelae [28]. The incidence rate was highest in very young infants, with a second peak observed in older adults (aged $>60$ years); the number of cases of MenW disease in adolescents was very low [21]. A mass vaccination campaign with a meningococcal quadrivalent vaccine was accordingly implemented by the Chilean Ministry of Health in October 2012 for children aged 9 months to 5 years, followed by the introduction of the meningococcal quadrivalent conjugate vaccine (MenACWY) into routine vaccination schedules at 12 months of age in 2014 [21, 28-31].

Similar to the experience in Chile, Argentina observed an increase in MenW disease prevalence around 2008, with the sequence type cc11 accounting for $78 \%$ of all MenW strains [9]. From 2012 to 2015, MenW was responsible for nearly half of all meningococcal disease cases across all ages, particularly among infants aged $<9$ months. In early 2017, Argentina introduced MenACWY into the national immunization plan for infants aged $>3$ months, with two doses at ages 3 and 


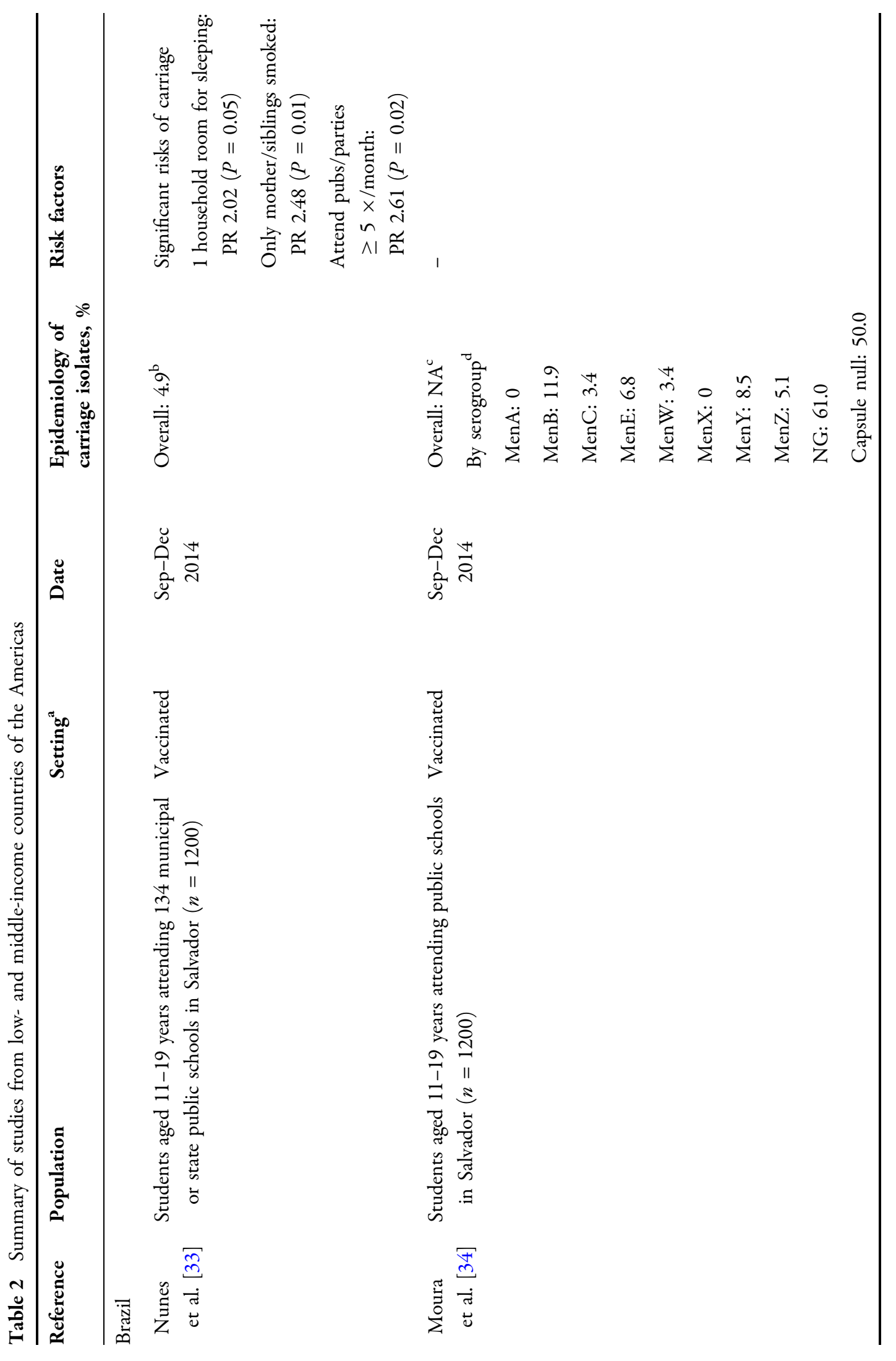




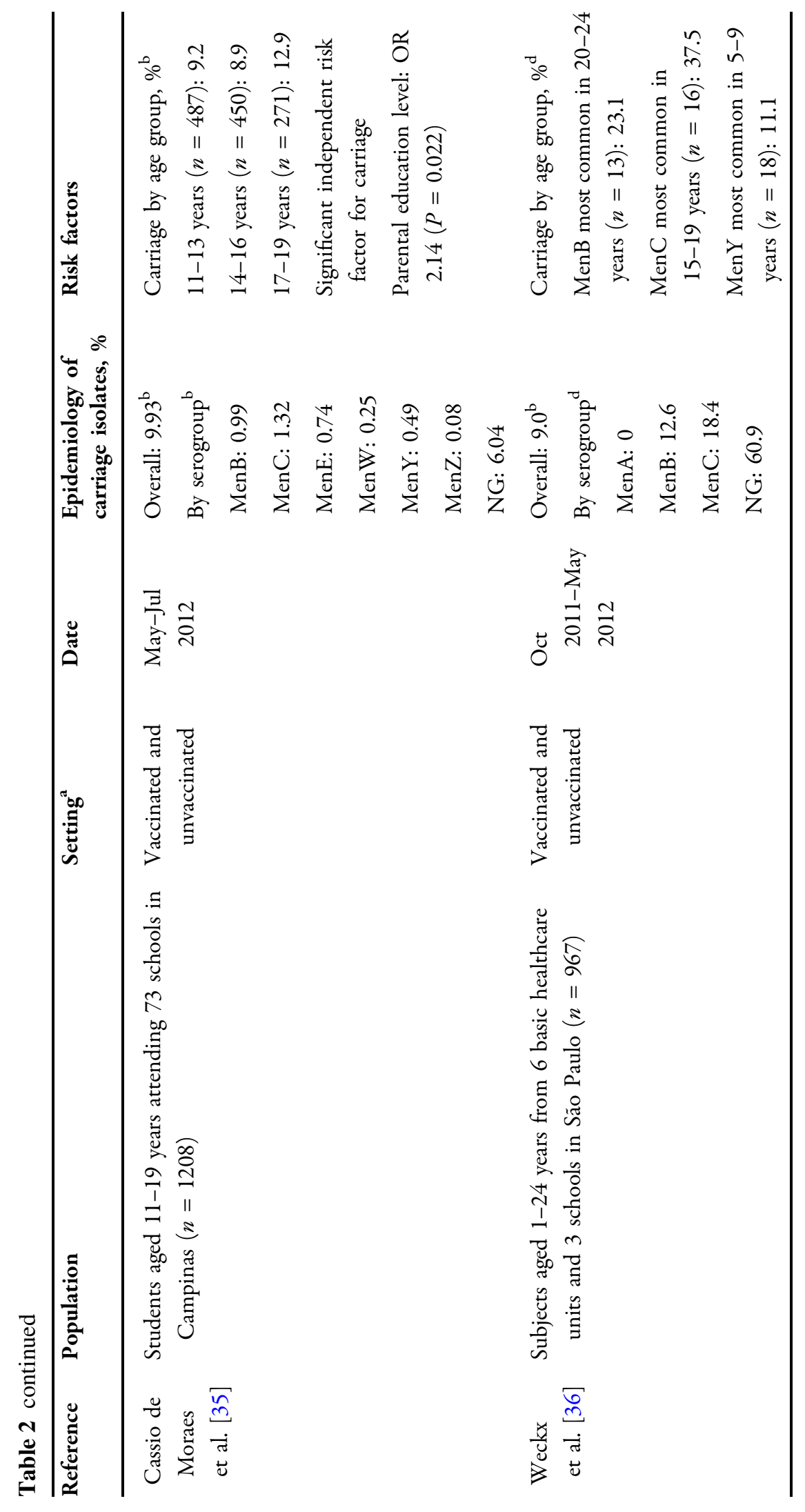




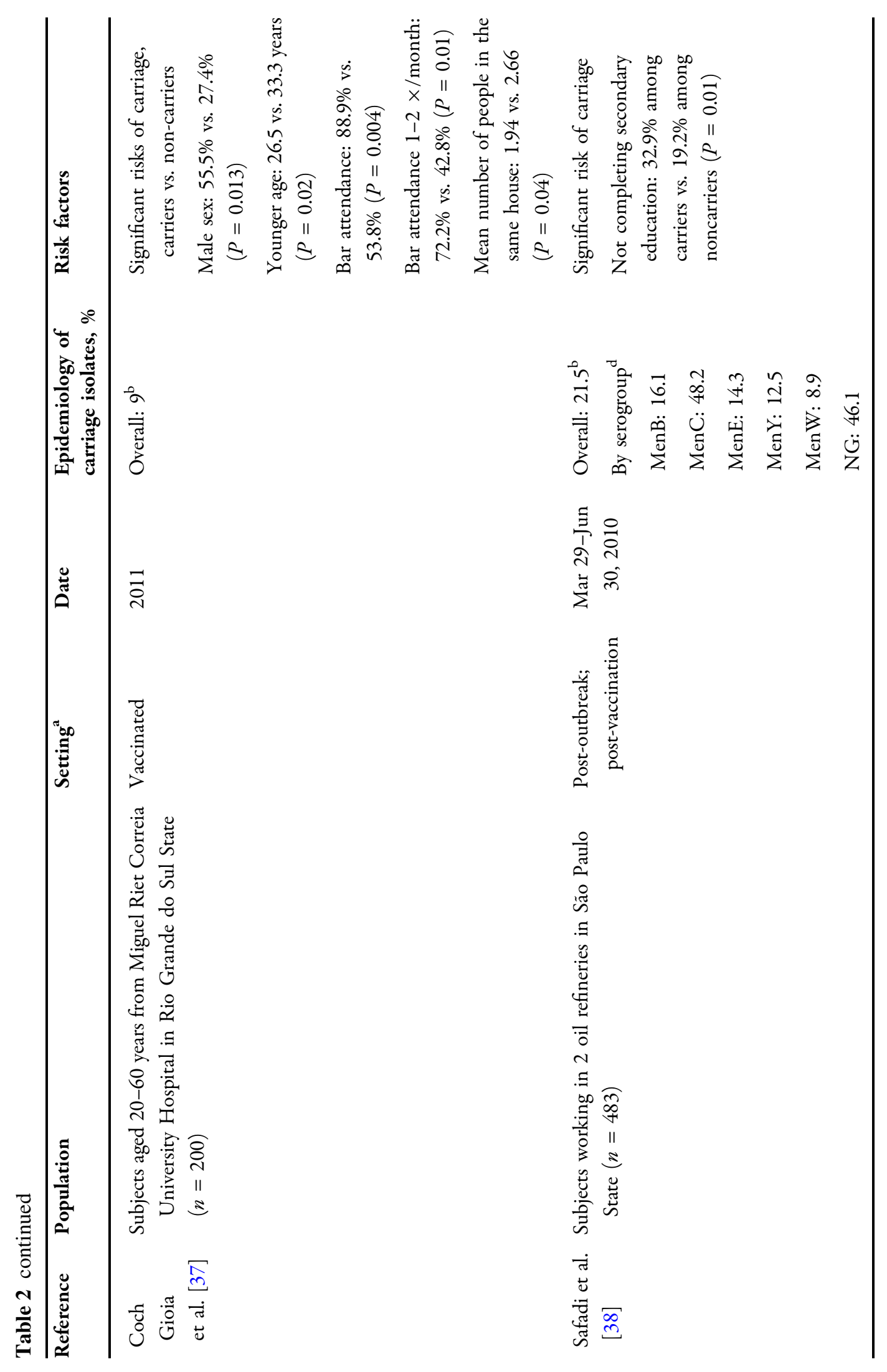




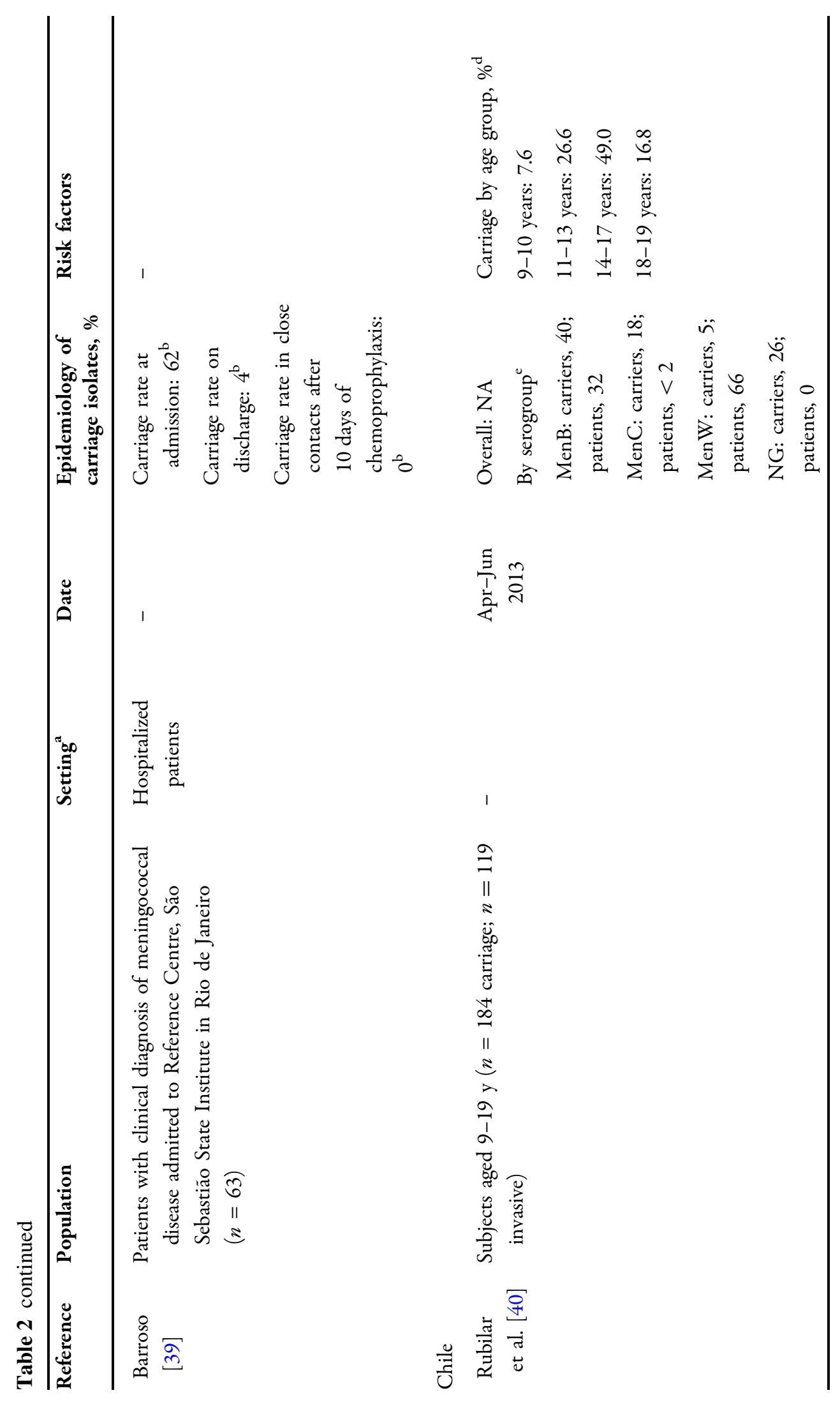




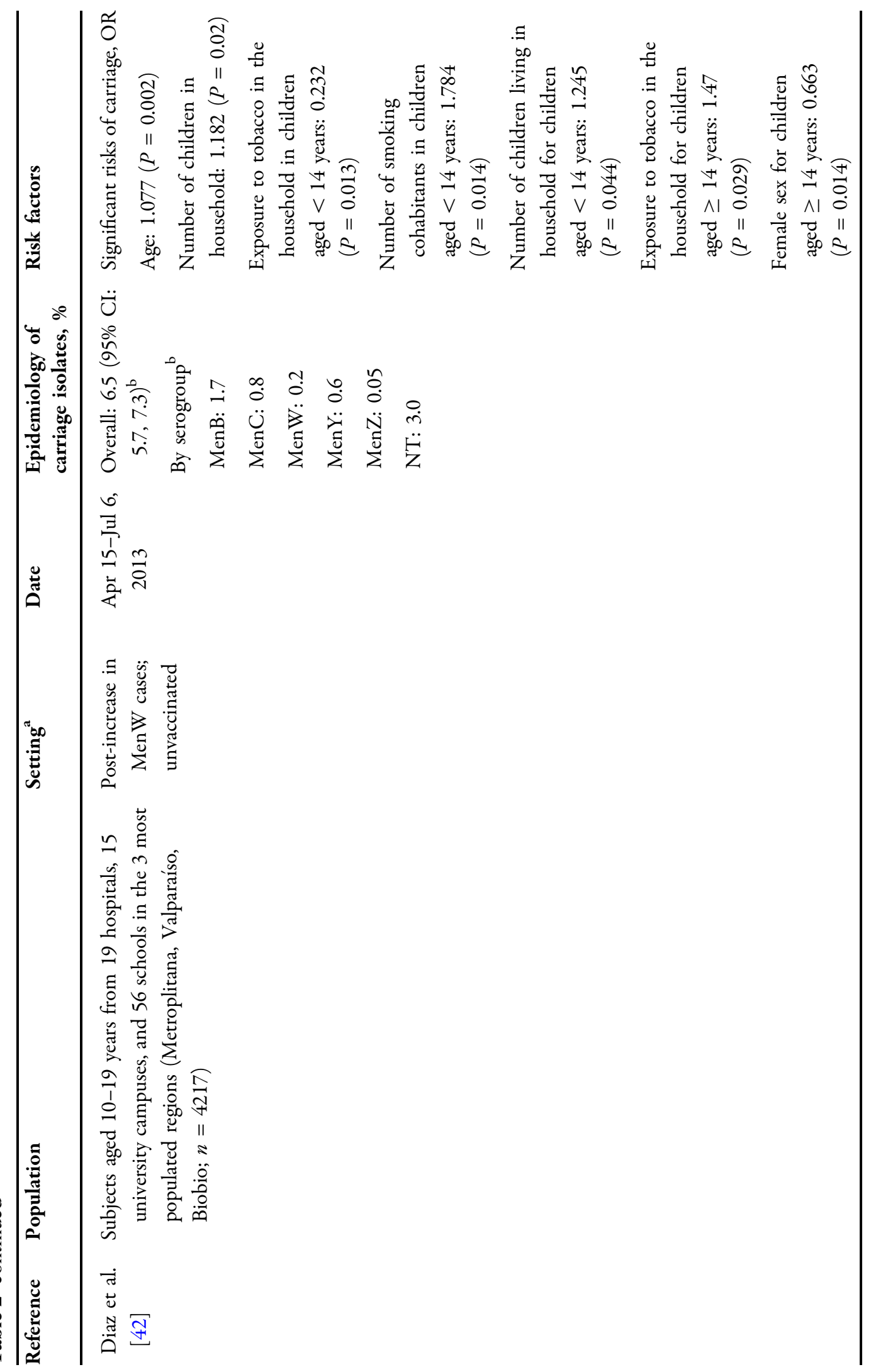




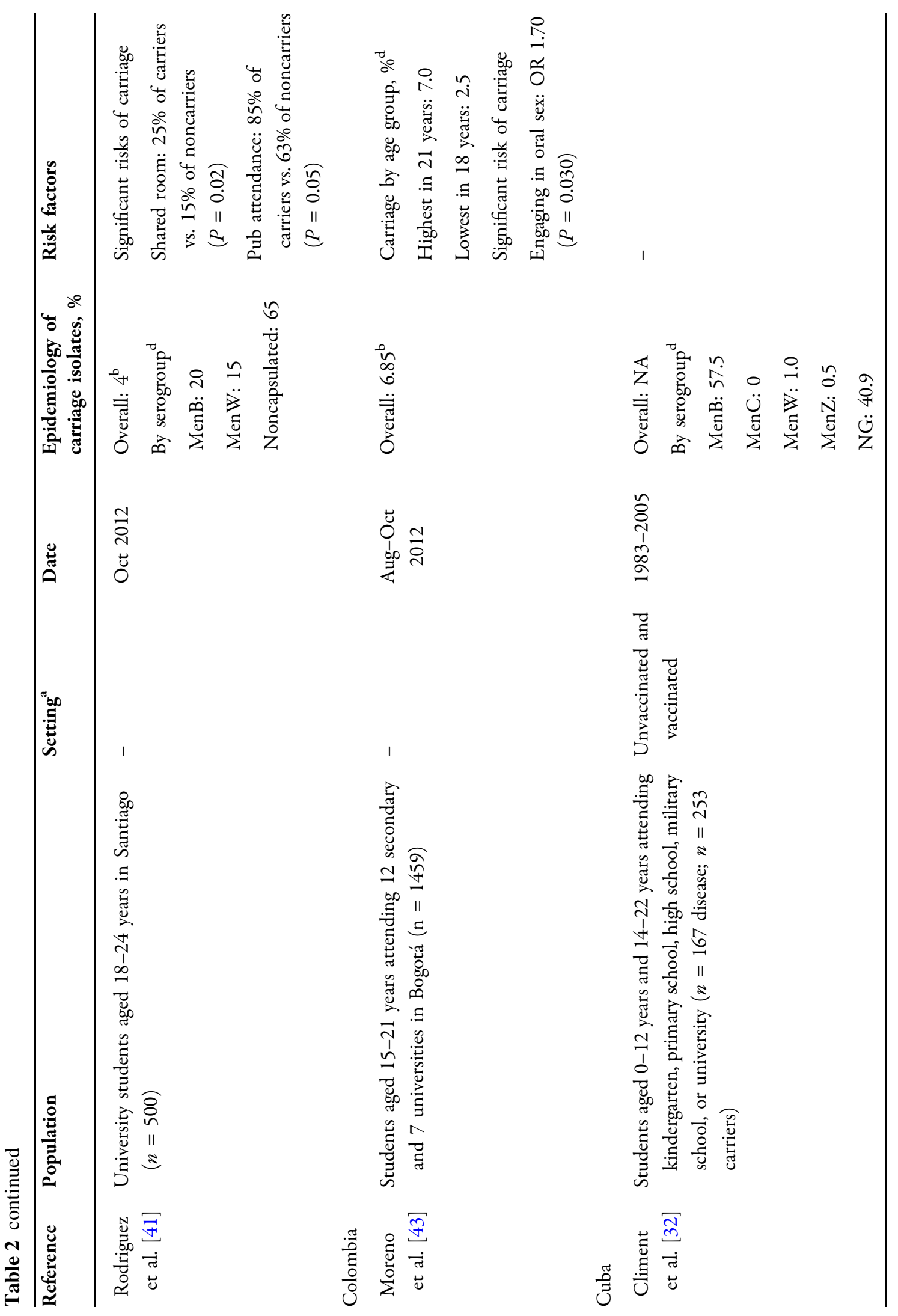




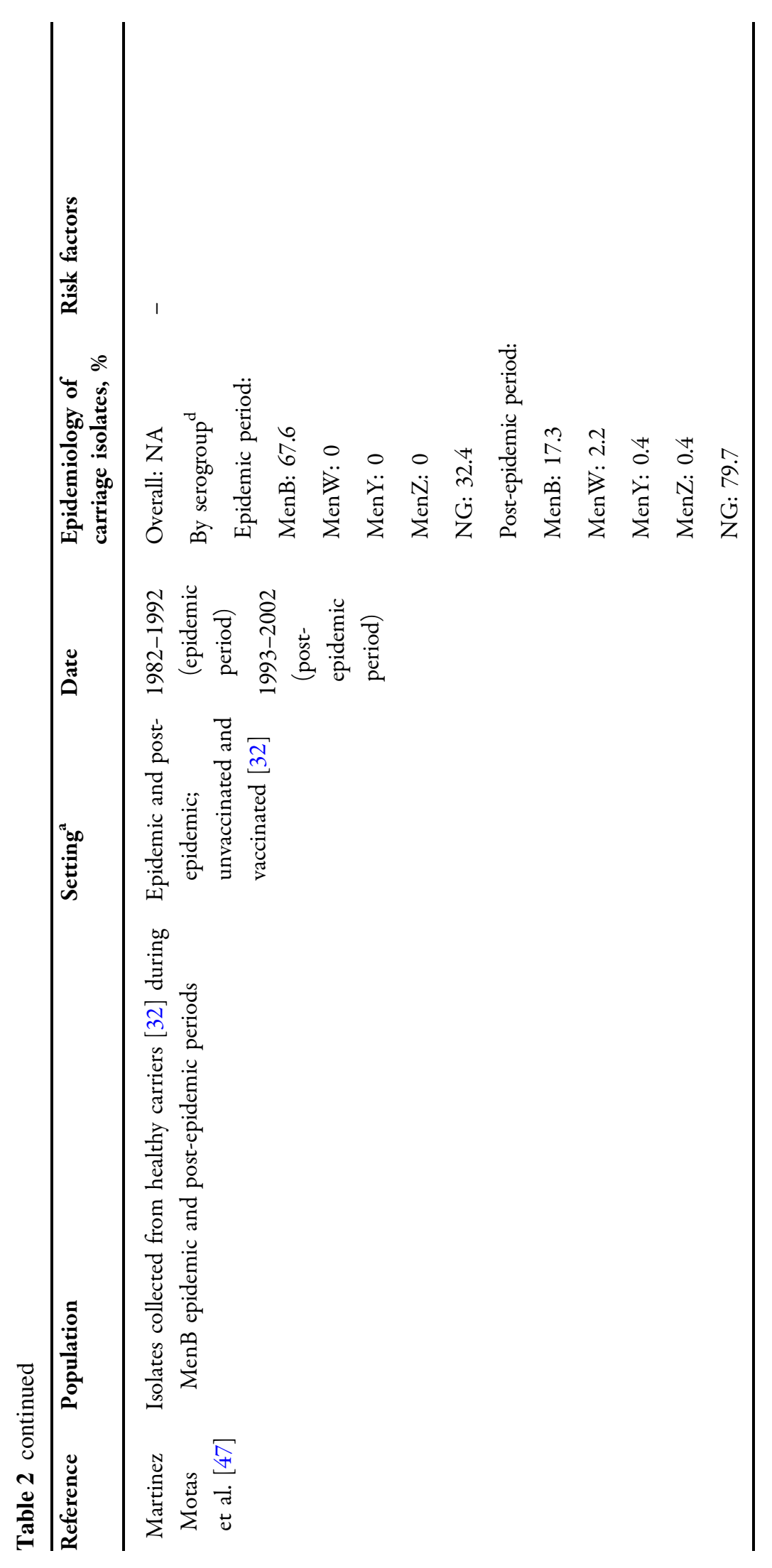




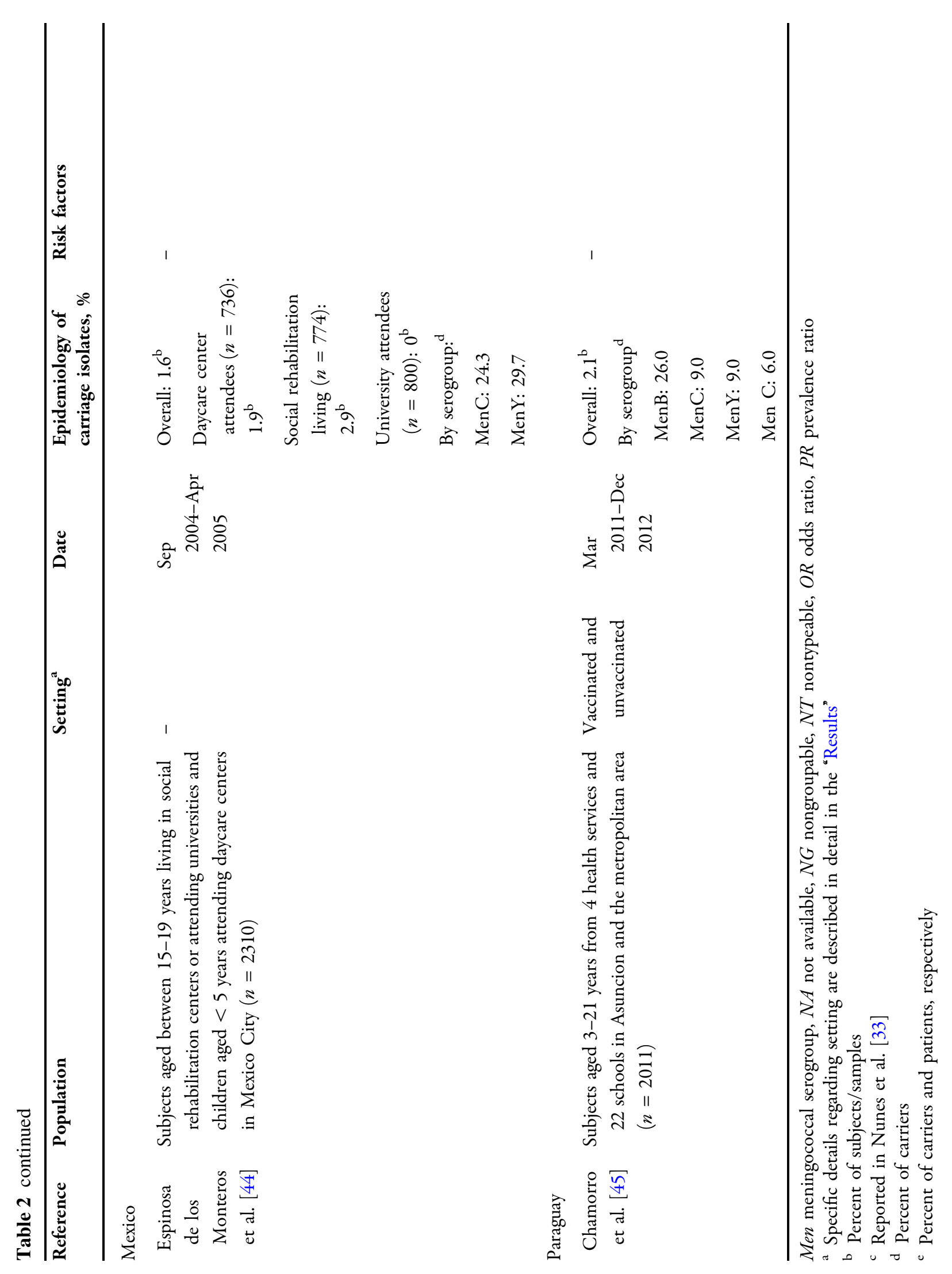


The Americas

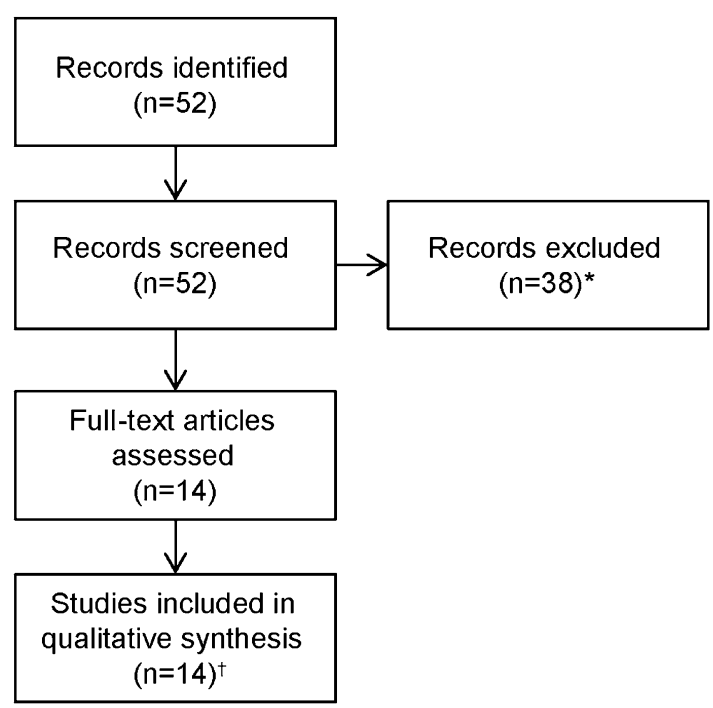

Fig. 1 Literature search results. Asterisk articles that did not meet the inclusion criteria or that fulfilled the exclusion criteria were excluded. Dagger one additional

5 months, respectively, followed by a booster at age 15 months. A single dose of the vaccine was also recommended for adolescents to reduce carriage rates. Surveillance of the impact of including MenACWY in the national immunization plan is still ongoing because of the program's recency.

In 1989, individuals aged 3 months to 24 years in Cuba were vaccinated with a combined MenB and MenC vaccine (VA-MENGOC$\mathrm{BC}$ ) because of an increased incidence of disease predominantly attributed to a single MenB clone (strain CU385) [32]. Importantly, vaccination resulted in a notable decrease in disease incidence from a peak of 14.4 cases per 100,000 population in 1983-1984 (before vaccine inclusion in the vaccination program) to $<0.4$ cases per 100,000 population in 2001 , and 0.1 cases per 100,000 population in 2008 .

\section{Literature Search Findings on Carriage in the Americas}

The literature search identified 52 publications from the Americas (Fig. 1). Of these, 14 met the inclusion criteria and were included in the review, including seven studies from Brazil [33-39], three from Chile [40-42], and one each
Asia

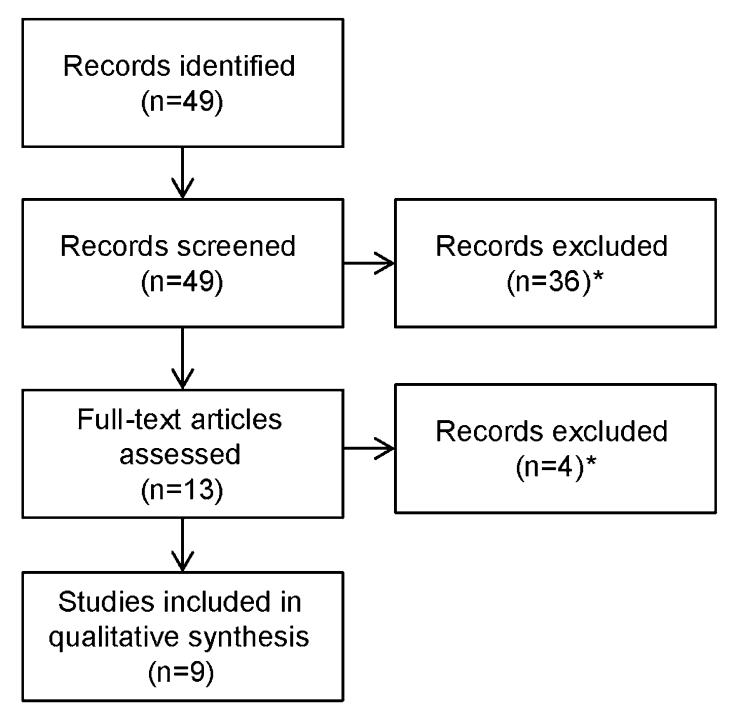

study from the authors' files was included for a total of 15 studies included in this analysis

from Colombia [43], Cuba [32], Mexico [44], and Paraguay [45] (Fig. 2; Table 2). An additional non-English language publication from Cuba from the authors' files was included [46]. No studies from Central America or the Caribbean were identified. Because different laboratory methods can affect carriage assessments, the methodology used to ascertain carriage and the source of the isolates within these studies have been summarized (Table S2).

\section{Brazil}

Seven studies reporting carriage in Brazil were identified. The most recent data were from two separate publications of the same sample. From September to December 2014, Nunes and colleagues assessed the meningococcal carriage of 1200 students aged 11-19 years enrolled in 134 municipal or state public schools in Salvador [33]. Participants were selected by a two-stage probabilistic method in which 155 schools were initially randomly selected and weighted according to the probability of school selection by the proportion of students in each of the schools in Salvador. The final stage was 


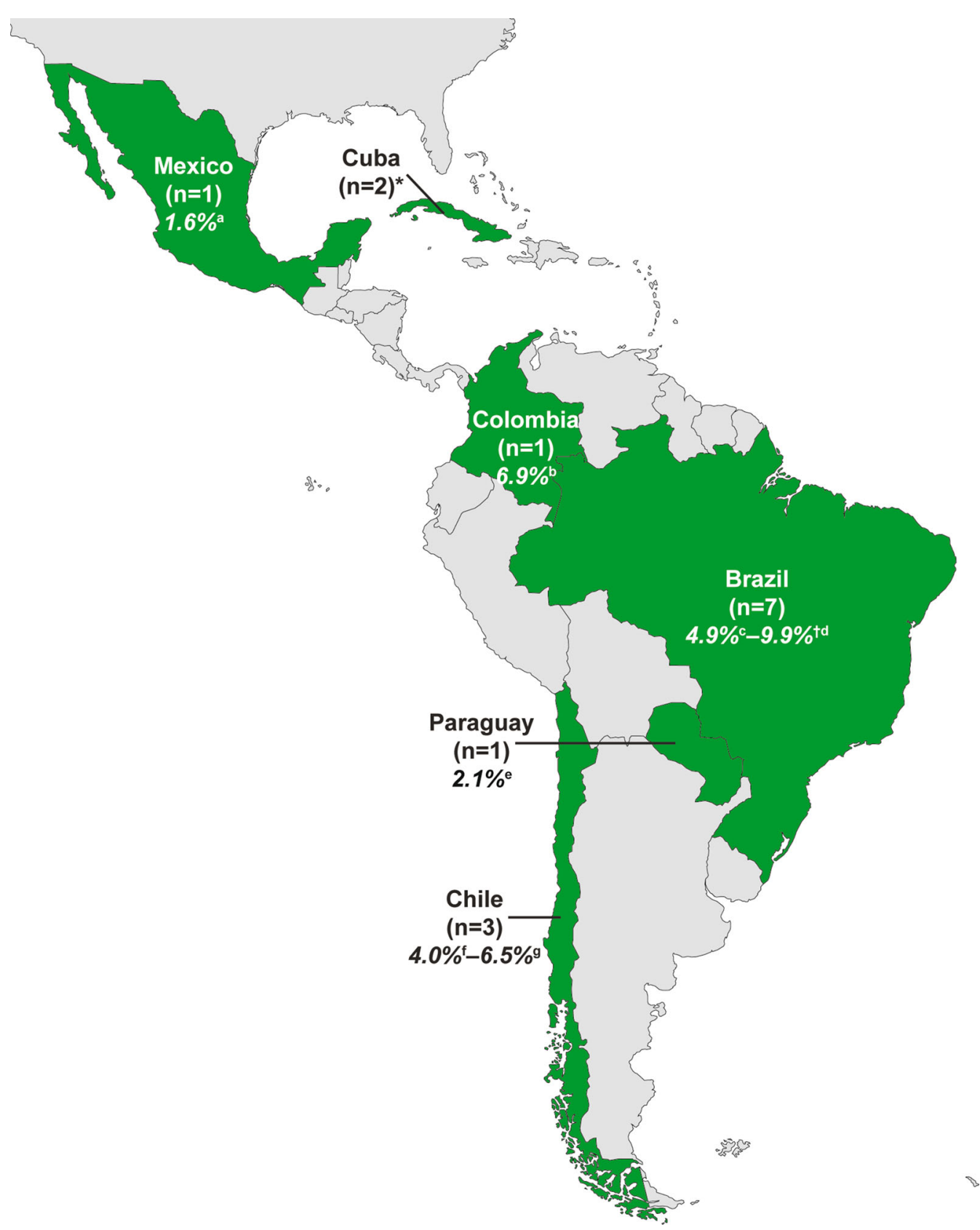

Fig. 2 Summary of literature search results and carriage rates by country in the Americas. Carriage rates are indicated in italics. Additional information on study design and population is provided in Table 2 and Table S2. Asterisk carriage rates not reported in identified study. Dagger does not include carriage rates from outbreaks or related to invasive meningococcal disease cases or their

selection of 134 of the 155 randomly selected schools that provided student names. The overall carriage rate was $4.9 \%$ (59/1200). contacts. Population age ranges: ${ }^{\mathrm{a}}<5$ years (attending daycare) and 15-19 years (living in social rehabilitation centers or students); b 15-21 years (students); ${ }^{\mathrm{c}}$ 11-19 years (students); ${ }^{\mathrm{d}}$ 11-19 years (students); ${ }^{\mathrm{e}} 3-21$ years (health center attendees and students); ${ }^{\mathrm{f}}$ 18-24 years (students); ${ }^{\mathrm{g}}$ 10-19 years (hospitals and schools)

Bivariate analysis found the following significant risks for carriage: having only 1 room for sleeping (2.02 prevalence ratio; $P=0.05)$, 
having only mothers who smoke (2.48 prevalence ratio; $P=0.01$ ), and attending a pub or party $\geq 5$ times per month (2.61 prevalence ratio; $P=0.02$ ). Multivariable analyses were not conducted. Moura and colleagues later characterized the 59 carriage isolates by serogroup and multilocus sequence typing (MLST) [34]. The majority of isolates were nongroupable $(61.0 \%$; $36 / 59)$. The most common groupable isolates were MenB (11.9\%; 7/59), MenY (8.5\%; 5/59), MenE $(6.8 \% ; 4 / 59)$, MenZ $(5.1 \% ; 3 / 59)$, MenC (3.4\%; 2/59), and MenW (3.4\%; 2/59); no MenA or MenX carriage isolates were identified. Of the 34 different sequence types identified, the most frequent were cc1136 (35.3\%; 12/34) and cc198 (32.4\%; 11/34). Hyperinvasive lineage complexes were also identified, the most common being cc23 (6.8\%; 4/59) and cc41/44 (5.1\%; $3 / 59)$. The authors postulated that the low rate of MenC carriage isolates was likely attributed to the mass MenC vaccination campaign for subjects aged 10-24 years conducted in Salvador in 2010, as described above.

Cassio de Moraes and colleagues assessed meningococcal carriage in 1208 students aged 11-19 years attending 73 schools in Campinas over a 3-month period (May-July, 2012) [35]. This period covers postimplementation of the MenC national immunization program in infants in 2010 described above. Public and private schools were randomly selected from the five health districts in Campinas according to the number of students in each grade corresponding to the age groups in the study. Participants from the selected schools were randomly chosen, and included up to five students from each class. The overall carriage rate in this sample was $9.9 \%(120 / 1208)$. The most common serogroups were MenC (13.4\%; 16/120), MenB (10.0\%; 12/120), MenE (7.5\%; 9/120), MenY (5.0\%; 6/120), MenW (2.5\%; $6 / 120)$, and MenZ (0.8\%; 1/120); the majority of isolates were nongroupable $(60.8 \% ; 73 / 120)$. By MLST, 36 sequence types were identified, with the most common being cc53 $(41.7 \% ; 15 / 36)$ and cc1136 (33.3\%; 12/36). Univariate analysis identified a significant increase in the percentage of carriers among type of school attended (11.3\% public school vs. $5.8 \%$ private school; $\left.\chi^{2}=7.834, \quad P=0.005\right), \quad$ recent influenza-like illness $\left(12.7 \%\right.$ yes vs. $8.5 \%$ no; $\chi^{2}=5.090$, $P=0.024)$, night club attendance $(13.8 \%$ yes vs. $5.8 \%$ no; $\left.\chi^{2}=15.768, \quad P<0.001\right), \quad$ passive smoking $\left(13.2 \%\right.$ yes vs. $8.4 \%$ no; $\chi^{2}=6.670$, $P=0.010)$, vaccination status with MenC polysaccharide or conjugate vaccine $(5.2 \%$ yes vs. $10.5 \%$ no; $\left.\chi^{2}=5.165, P=0.023\right)$, and parental level of educational attainment $(13.2 \%$ none vs. $10.3 \%$ fundamental vs. $10.4 \%$ basic vs. 5.6\% high; $\chi^{2}=8.787, P=0.032$ ). Multivariable analyses indicated that only parental education level, specifically a lower level of education, was a significant risk factor independently associated with carriage risk (odds ratio, 2.14; $P=0.022$ ).

From October 2011 to May 2012, Weckx and colleagues assessed carriage from a sample of 87 individuals aged 1-24 years recruited from six basic healthcare units and three schools in the Embu das Artes region of São Paulo; additional details regarding participant recruitment were not provided [36]. The study covered the period 10 months after introduction of the MenC vaccine in the national immunization program, as described above. The overall carriage rate was $9.0 \%(87 / 967)$, with higher rates in younger (10-14 years; $12.5 \%)$ and older adolescents (15-19 years; $12.6 \%$ ). Higher rates of carriage were observed among unvaccinated versus MenC-vaccinated subjects $(8.5 \%$ vs. $4.8 \%)$. The most common disease-associated serogroups were MenC (18.4\% of all N. meningitidis carriage isolates identified) and MenB (12.6\%); MenB carriage was most common in young adults (aged 20-24 years; 23.1\%), MenC in older adolescents (aged 15-19 years; 37.5\%), and MenY in children (aged 5-9 years; $11.1 \%$ ). The majority of carriage isolates were nongroupable $(60.9 \%)$, and no MenA carriage isolate was identified. Logistic regression analysis showed the number of household members was independently associated with increased risk of meningococcal carriage in the 15-19 years age group (odds ratio, $1.35 ; P=0.019$ ).

Coch Gioia and colleagues assessed oropharyngeal samples of 100 employees and 100 students aged 20-60 years from a university hospital in Rio Grande do Sul State in 2011, which also covered the period after inclusion of the MenC vaccine in the national 
immunization program [37]. The overall carriage rate was $9 \%(18 / 200)$. Carriage was significantly higher in men than women $(16.7 \%$ vs. $5.7 \% ; P=0.013)$. Other significant risk factors for carriage were bar attendance $(88.9 \%$ vs. $53.8 \%$ in carriers vs. noncarriers; $P=0.004$ ), bar attendance frequency $(72.2 \%$ vs. $42.8 \%$ for $1-2$ times monthly, $16.6 \%$ vs. $11.5 \%$ for $\geq 3$ times monthly; $P=0.01)$, mean age (26.5 vs. 33.3 years; $P=0.02$ ), and mean number of household members (1.94 vs. 2.66 ; $P=0.04)$; multivariable analyses were not described. Assessment of antibiotic sensitivity indicated that 13 of the carriage strains were sensitive to all tested antibiotics, and four were of intermediate sensitivity to penicillin and two to ampicillin.

MenC outbreaks occurred in two oil refineries in 2010 in São Paulo State, with a mass vaccination program with a combined MenA and MenC polysaccharide vaccine conducted in one of the refineries that achieved 91\% vaccine uptake [38]. Safadi and colleagues described meningococcal carriage in a sample of 483 workers from these two refineries from March 29 to June 30, 2010; the method of choosing the selected participants was not described [38]. The overall carriage rate was $21.5 \%(104 / 483)$, and the carriage rate was comparable in both refineries $(21.4 \%$ vs. $21.6 \%)$ even though the vast majority of workers in only one of the refineries had been vaccinated. Of the 56 serogrouped carriage samples, the most common was MenC (48.2\%; 27/56); less frequently observed serogroups were MenB (16.1\%; 9/56), MenE (14.3\%; 8/56), MenY (12.5\%; 7/56), and MenW (8.9\%; 5/56). Among the 27 isolates characterized by MLST (14 in the refinery that had received vaccination; 13 in the refinery that had not), the most common clonal complex was cc11 $(28.6 \% ; 4 / 14)$ in the refinery that had received vaccination and cc103 $(46.2 \% ; 6 / 13)$ in the refinery that had not. The only significant risk factor for carriage was not completing secondary education (32.9\% among carriers vs. $19.2 \%$ among noncarriers; $P=0.01$ ). The authors postulated that less educated workers in oil refineries were more likely to perform duties requiring use of ear protection that could force close contact during conversation and thereby facilitate meningococcal transmission.

Barroso and colleagues reported differences in carriage rates at hospital admission and after discharge for 55 patients with meningococcal disease as well as among close contacts who received chemoprophylaxis for 10 days [39]. The study was conducted at the São Sebastião State Institute in Rio de Janeiro over a period of 8 months, but the specific dates of the study were not reported. The carriage rate was $62 \%$ $(34 / 55)$ at admission and $4 \%(2 / 55)$ at discharge. No nasopharyngeal carriage isolates were identified in close contacts of patients.

\section{Chile}

Three studies from Chile assessed carriage. The most recently published was a study by Rubilar and colleagues on the correlation of IMD serogroups with meningococcal carriage serogroups in children and adolescents aged 9-19 years [40]. The study included 184 carriage samples from children and adolescents and 119 invasive samples from all ages obtained from the national reference laboratory. The carriage isolates were specifically obtained from three regions (Metropolitana, Valparaíso, and BioBío) from April to June 2013 (i.e., the period after the MenACWY vaccination program described above). It was not specified by the authors whether the sample included all carriage samples obtained during that period and from the three regions. MenB was the most common carriage isolate ( $40 \%$ of carriers) followed by nongroupable (26\%), MenC (18\%), and MenW (5\%). Although similar percentages of isolates between MenB carriage and disease isolates were found ( $40 \%$ vs. $32 \%)$, a similar effect was not observed for nongroupable (26\% vs. $0 \%$ ), MenC ( $18 \%$ vs. $2 \%)$, or MenW (5\% vs. $66 \%)$ isolates. Because of the emergence of the hypervirulent MenW cc11 strain within the country, the assessment of carriage and disease isolates by clonal complex was undertaken. It was found that MenW cc11 was predominant among disease isolates (66\%). However, carriage isolates demonstrated a higher diversity of clonal complexes, with cc41/44 being 
predominant and with only $5 \%$ of carriage isolates attributed to cc11.

Also, from Metropolitana, Valparaíso, and Bio-Bío in 2013 (April-July), Diaz and colleagues collected 4217 posterior pharynx specimens of children and adolescents aged 10-19 years from 19 hospitals as well as from 15 university campuses and 56 schools located close to the selected hospitals [42]. All individuals matching the selection criteria were asked to participate (i.e., healthy male and female subjects aged 10-19 years who provided consent to participate, had no history of IMD or chronic diseases, were not using immunosuppressants or antibiotics in the past month, and had not received previous meningococcal vaccination). The overall carriage rate was $6.5 \%(272 / 4217)$. The most prevalent serogroup was MenB (1.7\%) and nongroupable (3.0\%). The prevalence of MenC, MenW, MenY, and MenZ was $\leq 0.8 \%$. Univariate analysis found a significant association of carriage with age (mean 14.22 years for carriers vs. 13.77 years for noncarriers; $P=0.009$ ), number of intimate kissing partners in the past 30 days (mean 0.60 vs. 0.46; $P=0.044)$, and attending crowded events in closed spaces ( $49 \%$ vs. $37 \%$; $P=0.008)$. Multivariable regression analysis found a significant $7.7 \%$ increased risk of carriage per year of age $(P=0.002)$ and that the number of children in the household increased the risk by $18 \%$ per child $(P=0.02)$; however, no association of carriage with factors associated with overcrowding or geography was found. For children younger than 14 years, exposure to tobacco in the household was linked to a significantly decreased risk of carriage in multivariable regression analysis; however, a significantly increased risk of carriage was observed for those living in households with more smokers or more children. Conversely, individuals 14 years or older who were exposed to tobacco in the household had a significantly increased risk of carriage, and females had a significantly decreased risk of carriage.

The third study from Chile was by Rodriguez and colleagues and included 500 healthy university students in Santiago aged 18-24 years [41]. Participants who had previous meningococcal disease, chronic diseases, or reported antibiotic use in the previous month were excluded; other approaches to selecting participants were not described. The time the samples were collected (October 2012) corresponded with the increase in MenW cc11 cases described above. The overall carriage rate was $4 \%$ (20/ $500)$, with $20 \%(4 / 20)$ of carriage isolates being MenB, 15\% (3/20) MenW, and the rest being noncapsulated $(65 \% ; 13 / 20)$. Of the eight isolates in which a clonal complex could be assigned, the most commonly observed were cc41/44 (37.5\%; 3/8) and cc175 (25.0\%; 2/8). The only risk factor found to be significantly associated with carriage was sharing a room (25\% of carriers vs. $15 \%$ of noncarriers; $P=0.02) ;$ a trend for pub attendance in the previous month was observed as a risk factor for carriage ( $85 \%$ vs. $63 \% ; P=0.05)$. No statistically significant differences were seen between carriers and noncarriers in univariate analysis. Unlike the findings by Rubilar and colleagues [40], none of the MenW carriage isolates were attributed to cc11 [41].

\section{Colombia}

One study assessed meningococcal carriage in Colombia. Moreno and colleagues reported the prevalence of meningococcal carriage of students in Bogotá in a 3-month period in 2012 (August-October) [43]. All adolescents in the ninth to eleventh school grades were invited to participate, and those whose parents provided written consent for participation were included, as were consenting healthy university students. The study included 1459 students aged 1521 years from 12 secondary schools and seven universities. The overall carriage rate was $6.9 \%$ (100/1459); the highest carriage rates were in 20 (4.6\%) and 21 year olds (7.0\%) and the lowest in $18(2.5 \%)$ and 19 year olds (3.2\%). The most common serogroups were MenY (22\%; 22/100) and MenB (15\%; 15/100). MLST analysis of 74 isolates was undertaken; the most common clonal complexes were cc23 $(21.6 \% ; 16 / 74)$, cc53 (17.6\%; 13/74) and cc198 (13.5\%; 10/74). A Chi square analysis found a statistically significant difference in carriage among those who engaged in oral sex (odds ratio, 1.70; $P=0.030$ ), 


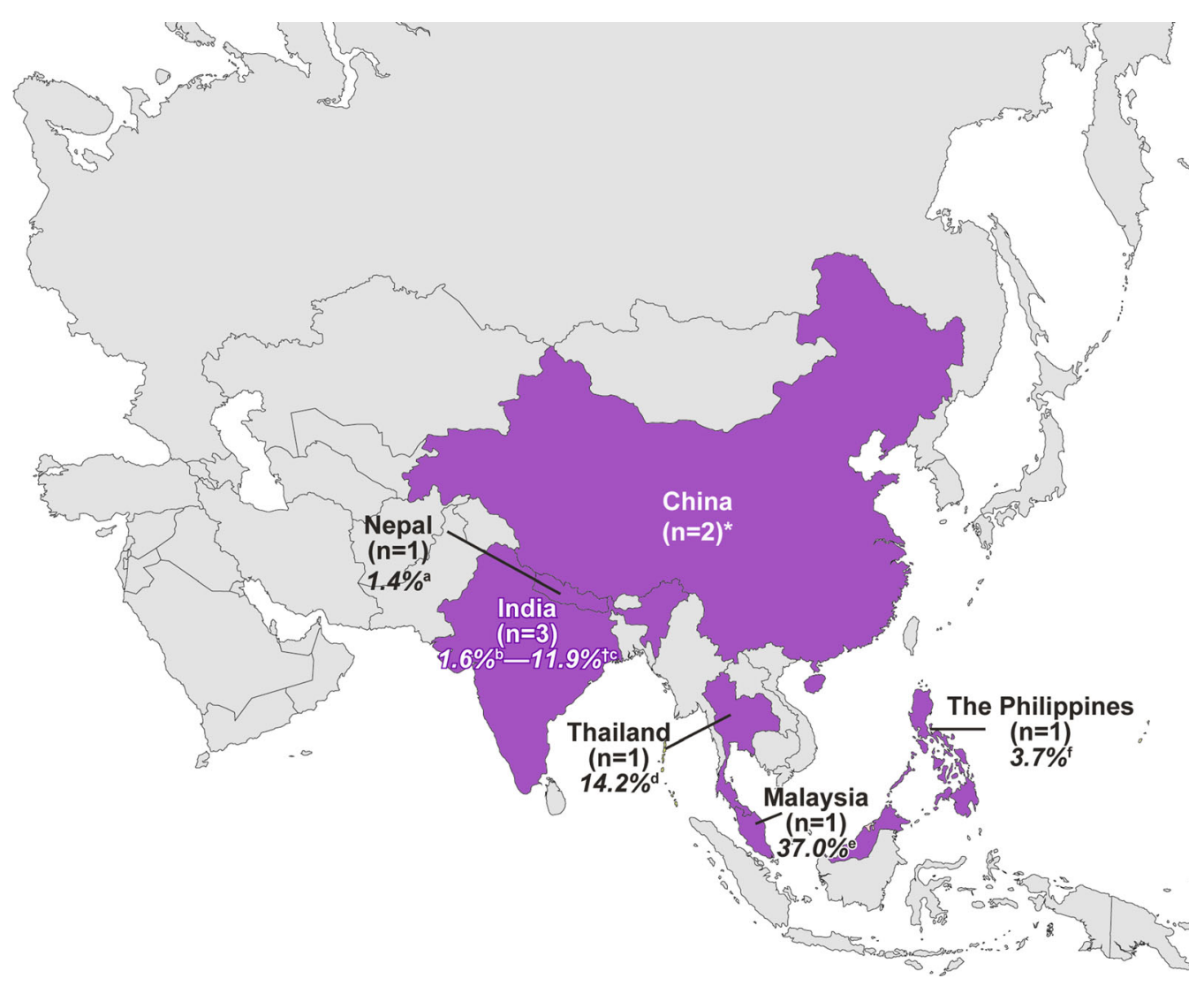

Fig. 3 Summary of literature search results and carriage rates by country in Asia. Carriage rates are indicated in italics. Additional information on study design and population is provided in Table 3 and Table S3. Asterisk identified studies did not report carriage rates outside of an outbreak setting. Dagger does not include carriage rates

but no significant effect of sex, intimate kissing, social venue attendance, cigarette smoke exposure, bedroom sharing, biomedical science students, respiratory infection, and antibiotic consumption in the previous month was found. Notably, a multilevel stratified analysis adjusted for sex, intimate kissing, social venue attendance, bedroom sharing, and smoking showed that the risk of carriage in those who engaged in oral sex was not significantly different from those who did not.

\section{Cuba}

One study by Climent and colleagues assessed the distribution of disease and carriage isolates related to invasive meningococcal disease cases or their contacts. Population age ranges, ${ }^{\mathrm{a}}$ 5-14 years (students); ${ }^{\mathrm{b}}$ 6-20 years (students); ${ }^{c} 16-25$ years (military recruits); ${ }^{\mathrm{d}}$ 5-15 years (students); ${ }^{\mathrm{e}} 17-24$ years (military recruits); ${ }^{\mathrm{f}}$ 5-24 years (students)

by meningococcal serogroups from Cuba between 1983 and 2005 [32]. The authors undertook the present analysis, which covered the periods before and after the introduction of VA-MENGOC-BC as described above, to understand the effect of the mass vaccination campaign on disease and carriage. Individuals receiving antimicrobial therapy in the past 7 days before sampling or receiving any immunomodulatory treatment were excluded. A total of 167 disease and 253 carriage samples from across Cuba were included, and the carriage isolates were obtained from healthy children aged $0-12$ years and from adolescents and young adults aged 14-22 years who were attending schools at the time of recruitment 
(i.e., kindergartens, primary schools, high schools, military schools, or universities). MenB accounted for $43.9 \%$ of carriage isolates (111/ 253), and no MenC carriage isolate was identified. Other carriage strains included nongroupable (31.2\%; 79/253), MenW (0.8\%; $2 / 253)$, and MenZ $(0.4 \% ; 1 / 253)$. Of the 63 sequence types identified by MLST, the most common was cc32 (11.1\%; 7/63). Notably, in the period after the epidemic, the specific MenB epidemic strain observed among carriage isolates significantly decreased, while strains not associated with epidemic outbreaks emerged.

A Spanish-language publication by Martinez Motas and colleagues that was obtained from the authors' files provides additional insights regarding the meningococcal isolates observed during and after the epidemic [47]. Among isolates collected in Cuba from healthy carriers during the epidemic period (1982-1992; $n=105$ isolates), MenB (67.6\%), serotype 4 (70.5\%), and subtype P1.19,15 (61.9\%) predominated [32, 47]. In contrast, during the postepidemic period following VA-MENGOCBC vaccination (1993-2002; $n=226$ isolates), nonserogroupable (NG; 79.7\%), nonserotypable (NT; 70.8\%), and nonserosubtypable (NST; $35.0 \%)$ strains were most common [47]. Overall, the predominant carriage phenotype during the epidemic was B:4:P1.19,15, while NG:NT:P1.NST was predominant in the postepidemic period.

\section{Mexico}

One publication assessed carriage in Mexico. Espinosa de los Monteros and colleagues reported the epidemiology of $N$. meningitidis carriage isolates in children younger than 5 years and adolescents in Mexico City from September 2004 to April 2005 [44]. Using a nonprobabilistic group sampling method from the healthy population, investigators studied children attending daycare centers $(n=736)$ and adolescents in social rehabilitation centers $(n=774)$ or attending universities $(n=800)$; 2310 nasopharynx swabs were obtained from these individuals. The overall carriage rate was $1.6 \%(37 / 2310)$, with the greatest rate observed in adolescents living in social rehabilitation centers $(2.9 \% ; 23 / 774)$ and in children attending daycare centers $(1.9 \% ; 14 / 736)$. No carriage isolates were identified from university students. The most frequent carriage serogroups were MenY (29.7\%), MenC (24.3\%), and MenB (10.8\%). Nontypeable isolates accounted for $27.0 \%$ of carriage isolates.

\section{Paraguay}

One study evaluated carriage in Paraguay in individuals aged 3-21 years between March 2011 and December 2012 [45]. Children were recruited from four health services and individuals aged 6-21 years were recruited from schools and universities from Asunción and the surrounding areas. A total of 2011 oropharyngeal swabs were obtained. The overall carriage rate was $2.1 \%$, with no significant differences demonstrated by sex, age group, or area of residence. Genogrouping revealed that $26.0 \%$ of strains were MenB, 9.0\% were MenW, 6.0\% were MenY and $6.0 \%$ were MenC. Overall, $61.9 \%$ of carriage isolates were nontypeable. The small sample size precluded inference of risk for meningococcal carriage (i.e., close habitation, respiratory infection, and smoking and vaccination status).

\section{Asia}

\section{Surveillance, Disease, and Vaccination}

Currently, low and middle income countries in Asia do not have routine and robust IMD surveillance programs; therefore, disease burden and at-risk populations are not well elucidated $[19,48]$. In addition, few countries in the region currently include meningococcal vaccines in their national immunization programs (Table S1) [24].

Because of limited data, the epidemiology of IMD in Asia is uncertain, although the disease burden is unlikely to be large due to the nature of meningococcal disease. It appears that MenA and MenC may predominate, although reports of MenW disease have become more frequent in several countries [49]. A total of 292 IMD cases were reported in Taiwan during 1996-2002, 
with the majority of confirmed cases due to MenB and MenW [50]. MenA was the predominant cause of IMD in China from the 1950s to the 1980s [51], but after the introduction of MenA vaccination using a polysaccharide vaccine, an increase in MenC disease was observed $[49,51]$. More recently, cases due to MenB and MenW have also increased [49]. Although reported cases of meningitis due to IMD have decreased in India since 2002, the number of reported IMD outbreaks has increased; since 2005 there have been three significant outbreaks, all caused by MenA [49, 52]. In the Philippines, data collected during 2008-2013 showed an increase in cases of meningococcemia (73 and 182 cases in 2008 and 2013, respectively); these cases most commonly occurred in infants and young children and were mostly due to MenA [49].

Literature Search Findings on Carriage in Asia The literature search identified 49 publications from Asia (Fig. 1). Of these, nine met the inclusion criteria and were included in the review, including two studies from China $[51,53]$, three from India [54-56], and one each from Malaysia [57], Nepal [58], the Philippines [48], and Thailand [59] (Fig. 3; Table 3). Because different laboratory methods can affect carriage assessments, the methodology used to ascertain carriage within these studies is summarized (Table S3).

\section{China}

Two studies from China assessed meningococcal carriage. The first study was conducted in Jinan City in 2010 at the time of a MenC disease outbreak in three young adult male prisoners. Zhang and colleagues determined the point prevalence of meningococcal carriage in 166 fellow prisoners, 16 of whom shared the same cell as the IMD patients [53]. N. meningitidis was isolated from pharyngeal swabs of 10 of the 16 cellmates (carriage rate $62.5 \%$ ). These isolates included six MenC, one MenB, one MenW, and two nongroupable strains. Of the 150 other fellow inmates sampled, 37 were found to be carriers, with isolates including
23 MenC, nine MenB, two MenA, one MenY, and two nongroupable strains (carriage rate $24.6 \%$ ). The carriage rate was significantly higher in cellmates of the patients compared with non-cellmates, both over all strains $(P<0.01)$ and for the MenC cc4821 clone associated with the outbreak (37.5\% vs. $13.3 \% ; P<0.05$ ).

In the second study, Zhou and colleagues analyzed 241 meningococcal strains isolated from throat swabs of asymptomatic carriers across 22 provinces in China during 2003-2008 (these provinces have laboratories that routinely collect $N$. meningitidis isolates). The analysis revealed a predominance of MenC $(62.2 \%)$, MenB (19.5\%), and MenA isolates (9.5\%), low frequencies of MenW, MenZ, MenX, and Men29E isolates ( $\leq 1.2 \%$ each), and a moderate frequency of nongroupable isolates (6.2\%) [51]. The study also characterized 130 disease isolates collected during the same time period; of these, $58.5 \%$ were MenC and $34.6 \%$ were MenA, whereas MenB accounted for only $3.9 \%$. cc5 and cc4821 were the most commonly occurring clonal complexes in both disease and carriage isolates, cc5 being associated with most MenA isolates (94.1\%) and cc4821 with most MenC isolates (87.6\%). However, because some of the carriers in this study were associates of IMD cases, the study's findings may not accurately represent normal carriage.

\section{India}

Three studies from India assessed carriage. The most recently published was a meningococcal carriage study by Jha and colleagues conducted in 1995 in military recruits in India which investigated a range of risk factors potentially associated with carriage [54]. The overall carriage rate in throat swabs obtained over a 3-month period from 360 new recruits aged $16-25$ years was $11.9 \%$. The highest carriage rate was observed among recruits from the eastern region $(18.0 \%)$ and the lowest was among those from the central region $(4.7 \%)$ of the country. A significantly higher $(P<0.05)$ carriage frequency was identified in recruits from joint families (14.4\%) compared with 
those from nuclear families (7.4\%); it was suggested that this difference may be because of the overcrowded living conditions frequently associated with a joint family, and further noted that this family structure is more common among lower socioeconomic classes in India. Although carriage rate increased with age and was higher among recruits previously living in mud houses compared with those previously living in concrete houses $(15.2 \%$ vs. 9.8\%), neither of these associations was statistically significant. Religion, education, level of personal hygiene, smoking, barrack type, upper respiratory tract infection, and previous tonsillectomy also showed no significant association with the carriage state.

The second study from India was by Ichhpujani and colleagues, who, during January 1986 to December 1987, collected nasopharyngeal samples from 6513 healthy students aged 6-20 years living in various locations across Delhi [55]. Most of the students sampled belonged to middle or lower income strata; further clarification regarding the participant selection was not provided. Overall, N. meningitidis was isolated from 1.6\% (107/6513) of subjects, with no observed differences in carriage rate by age or sex. During the same time period, $4.7 \%(557 / 11870)$ of pyogenic cerebrospinal fluid samples processed in six sentinel laboratories in Delhi were positive for $N$. meningitidis. All carriage and disease isolates were MenA, although no correlation between carriage rates and disease prevalence was identified.

A third study carried out in India examined the carriage rate among contacts of 11 index patients diagnosed with invasive MenA disease during March to June 1985 in Delhi [56]. Paul and colleagues evaluated 272 contacts, 53 of whom were family/household contacts and 219 of whom were neighbors or schoolmates of an index case. Nasopharyngeal swabs were initially obtained from contacts within $96 \mathrm{~h}$ of the index patient being admitted to the hospital; in the second phase of the study, 130 contacts with initially negative cultures were resampled 24 weeks later. In total, 402 swabs from 272 contacts were processed. Individuals older than 14 years made up $56.2 \%$ of contacts, and just over half were male $(54.4 \%)$. None of the contacts sampled had received previous chemoprophylaxis or vaccination against meningococcal disease. N. meningitidis was cultured from three of the 272 initial swabs; all three isolates were MenA strains and collected from family/household contacts. Subsequently, the carriage rate at the time of the first sampling was just $1.1 \%(3 / 272)$ among all contacts and $5.7 \%(3 / 53)$ among family/household contacts. Of the 130 repeat swabs, two cultured positive (MenA and MenC, respectively); both originated from nonfamily/household contacts and likely represented ongoing community transmission. All identified carriers were younger than 13 years and asymptomatic; $80 \%(4 / 5)$ were male.

\section{Malaysia}

One study from Malaysia assessed meningococcal carriage. This survey by Rohani and colleagues was conducted in June 2005 and investigated meningococcal carriage in military recruits during the third month of residence and intensive training at an army camp [57]. A total of 3195 healthy recruits aged $17-24$ years were sampled, and both throat and nasal samples were collected from each recruit. $N$. meningitidis was isolated from 1181 subjects, giving an overall carriage rate of $37.0 \%$ (1181/ 3195). Among female subjects, $19.7 \%$ were carriers; among male subjects, $39.2 \%$ were carriers. Of the 1181 strains isolated, 210 were randomly selected for serotyping and 100 were tested for antibiotic susceptibility. Of the serotyped strains, $81.0 \%$ were MenX, MenY, or MenZ; $4.7 \%$ were MenW; and $3.3 \%$ were MenA. The remaining $11.0 \%$ could not be typed due to autoagglutination (4.7\%), lack of reactivity with the available antisera (5.2\%; available antisera were to serogroups A, B, C, D, W, X, Y, and Z), or cross-reactivity with the available antisera $(0.9 \%)$. Of the isolates examined for antibiotic susceptibility, all were susceptible to chloramphenicol, rifampin, levofloxacin, and cefotaxime; $84 \%$ were resistant to cotrimoxazole. Although $12.5 \%$ of tested isolates showed reduced susceptibility to penicillin in the agar 
disc diffusion test, with minimum inhibitory concentrations (MICs) between $0.023 \mu \mathrm{g} / \mathrm{mL}$ and $0.064 \mu \mathrm{g} / \mathrm{mL}$, all isolates still fell within the susceptible category based on the MIC of $\leq 0.064 \mu \mathrm{g} / \mathrm{mL}$.

\section{Nepal}

The prevalence of a carriage state for a range of bacterial respiratory pathogens was evaluated in school children in Pokhara during 2015 as reported by Thapa and colleagues [58]. The 204 healthy children younger than 15 years who were present on the day of sample collection were enrolled in the study. There were equal numbers of students from private and government schools ( $n=102$ students each); 52 participants from private schools resided in school hostels and were aged 10-14 years. No participant had received meningococcal vaccination. Swabs taken from the posterior pharyngeal wall and tonsils of each subject were cultured for a range of bacterial pathogens, including $N$. meningitidis. Among the 204 students sampled, three were $N$ meningitidis carriers, giving an overall carriage rate of $1.4 \%$. Of these three carriers, two were aged 5-9 years (carriage rate, $3.7 \% ; 2 / 54$ ) and the other was aged $10-14$ years (carriage rate, $0.6 \% ; 1 / 150$ ); all had a history of repeated throat infection. Antibiotic sensitivity testing revealed that the three meningococcal isolates were resistant to penicillin. Serotyping and molecular characterization of isolates were not undertaken.

\section{The Philippines}

In a cross-sectional meningococcal carriage study conducted in the Philippines by Gonzales and colleagues, posterior pharyngeal swabs were collected between August 2013 and March 2014 from healthy individuals aged 5-24 years [48]. Individuals who had previously received meningococcal conjugate vaccination were excluded from the study. Subjects were sampled across three sites in Manila (1 elementary school, 1 high school, and 1 university) using a stratified random sampling method (i.e., school year was used as the stratum). Subjects were then stratified into four age groups: 5-9 years, $n=248 ; \quad 10-14$ years, $n=210 ; \quad 15-19$ years, $n=232$; and $20-24$ years, $n=247$. Among all age groups, $43.2 \%$ of subjects were male and $97.4 \%$ were nonsmokers. Of the 937 subjects, 35 were found to be carriers of $N$. meningitidis, giving an overall carriage rate of $3.7 \%$ (95\% CI: $2.6,5.2)$. Of the 35 carriers, 28 carried a single meningococcal serogroup (MenB, $n=23$; MenC, $n=3$; MenY, $n=2$ ), two carried multiple serogroups (MenY/MenW, $n=1$; MenB/MenY/ MenW, $n=1$ ), and five carried strains for which a capsular serogroup was not identified. Clonal complex was determined for 31 isolates, the most common being cc $175 \mathrm{C}$, cc $41 / 44 \mathrm{C} / \mathrm{L} 3$, and cc5656; $68 \%$ of the identified complexes mirrored isolates from IMD cases reported in Europe during 2012. The prevalence of carriage varied by age and was highest in the 10- to 14-year age group (carriage rate, 9\%; 95\% CI: $5.5,13.8 ; P<0.001)$. Carriage was also found to be weakly associated with having a larger number of siblings $(P=0.08)$, whereas no significant relationship was apparent with sex, type of living accommodation, smoking, or number of household inhabitants was apparent. The authors concluded that individuals aged 10-14 years may play an important part in meningococcal transmission in the Philippines.

\section{Thailand}

A study carried out by Danchiavijitr and colleagues during late 1983 and early 1984 assessed $N$. meningitidis carriage in children attending three primary schools in Nakhon Chaisi District, $40 \mathrm{~km}$ west of Bangkok [59]. Subjects were from a medium socioeconomic class and were likely representative of children living in central Thailand. Pharyngeal swabs were collected from 843 healthy children aged 5-15 years, 431 of whom were male. The overall carriage rate was $14.2 \%$. When carriage was assessed according to age, rates were highest in 14 and 15 year olds; however, low numbers of subjects of these ages ( $n=12$ and $n=1$, respectively) limited the relevance of this finding. A total of 94 strains were tested for antibiotic sensitivity: all were sensitive to chloramphenicol, erythromycin, and 


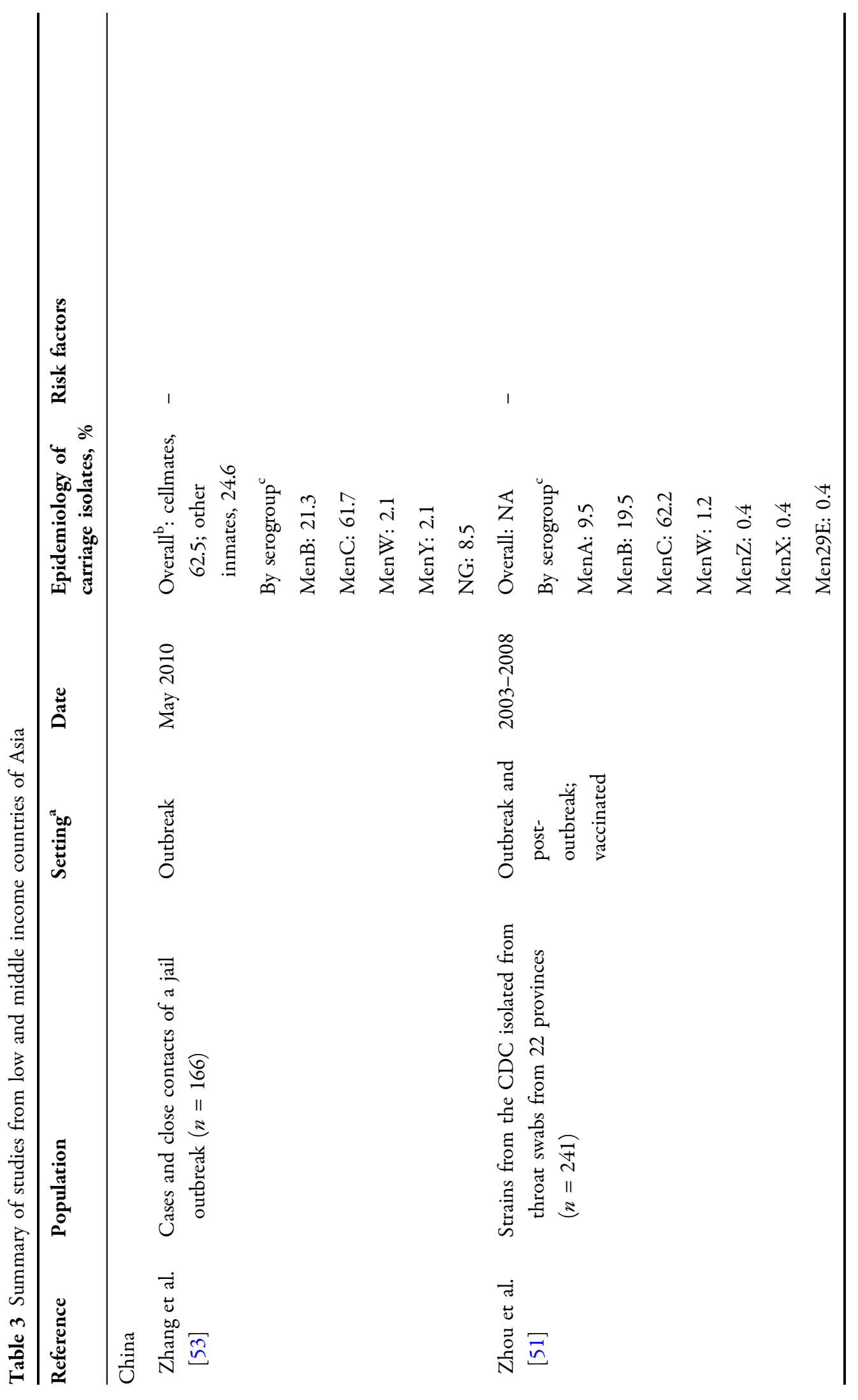




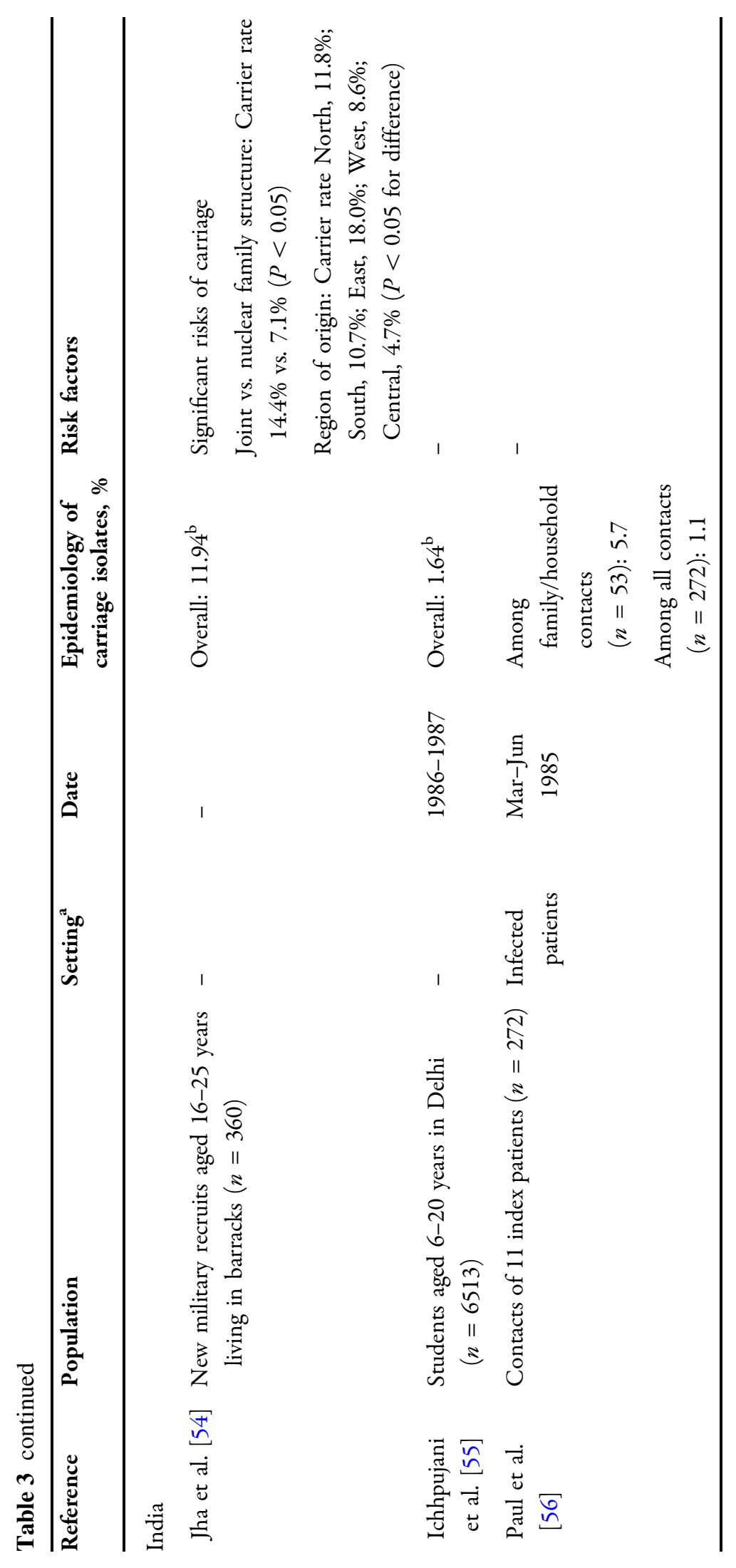




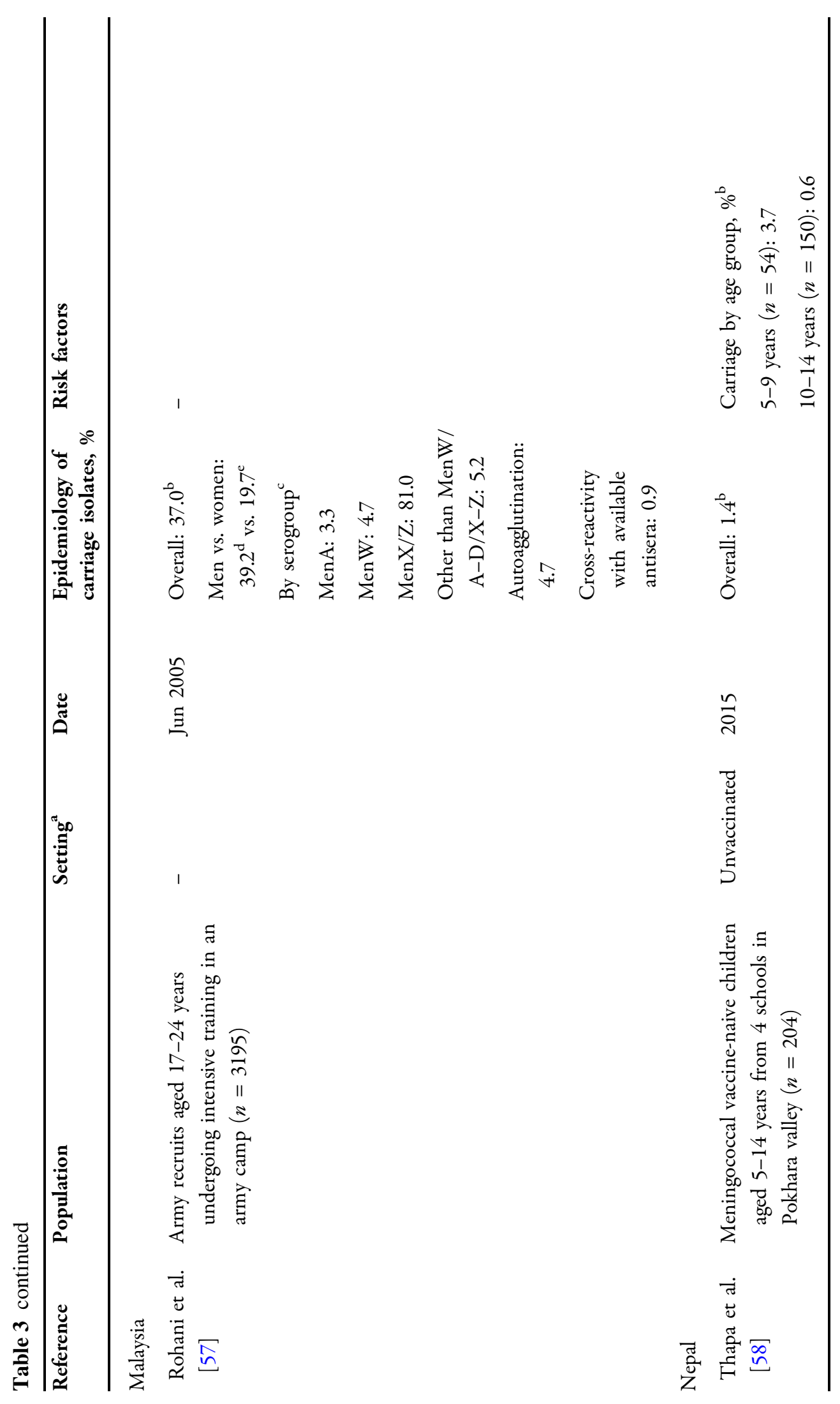




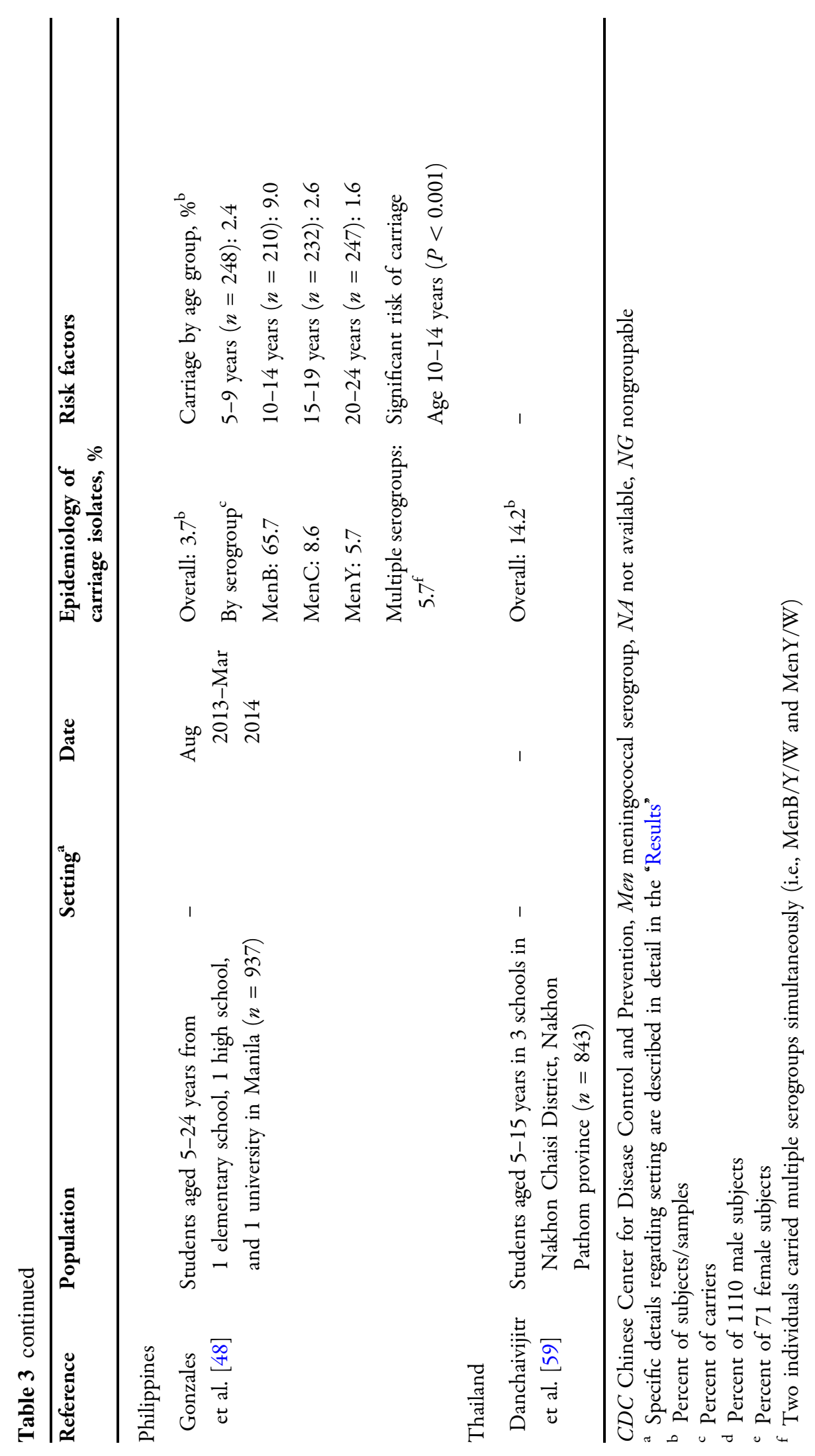


minocycline; 92 were sensitive and two were intermediately sensitive to penicillin; four were resistant to rifampin; and high levels of resistance to sulfadiazine and cotrimoxazole were apparent $(43.6 \%$ and $58.5 \%$ of strains, respectively). The authors commented that the carriage rate observed in the study did not appear to reflect the very low rate of reported meningococcal meningitis in Thailand at the time [59].

\section{DISCUSSION}

As demonstrated by our analysis, very limited published data are available on $N$. meningitidis carriage in low and middle income countries of the Americas and Asia. Of the published data identified, there was a notable lack of extensive contemporary information and no standardization of sampling or laboratory methods. Moreover, the preponderance of data was in children and adolescents from small populations, often from limited areas in a specific country. Many countries in these regions do not have any published data on meningococcal carriage.

The overall carriage rate in low and middle income countries of the Americas ranged from 1.6\% [children (2-71 months) and adolescents (15-19 years) in Mexico City in 2004 to 2005] [44] to 9.9\% (students aged 11-19 years in Campinas, Brazil in 2012) (Fig. 2) [35]. The carriage rate among close contacts of IMD patients following an outbreak was much higher (21.5\%) [38]. In low and middle income countries of Asia, the carriage rate varied from $1.4 \%$ (children aged 5-14 years in 2015 in Nepal) [58] to $14.2 \%$ (children aged 5-15 years in central Thailand in 1983-1984) (Fig. 3) [59]. In military recruits, the carriage rate ranged from $11.9 \%$ (aged 16-25 years in India) [54] to $37 \%$ (aged 17-24 years in Malaysia in 2005) [57]. There was also an inconsistent rate of carriage among contacts of patients with IMD in Asia, reflecting the diverse populations sampled, ranging from $62.5 \%$ for cellmates of patients with IMD in a Chinese prison [53] to $5.7 \%$ among household/family members of patients in India [56]. Of note, the comparison of data between countries should be undertaken with caution because there were notable differences between studies that could affect results, including populations assessed, sampling techniques (Tables S2, S3), time periods, and standard surveillance activities employed in each country or region. Because of these differences, we were not able to perform comprehensive analyses of meningococcal carriage by age in low and middle income countries of the Americas and Asia. Future carriage studies that follow a controlled or structured design are thus critical for defining carriage characteristics in these regions and allowing comparisons to high income countries such as the United Kingdom [13].

Some of the studies in our review assessed the antibiotic susceptibility of carriage isolates. In the study from Nepal, all three isolates were found to be resistant to penicillin [58]. In contrast, studies from Brazil [60], Thailand [59], and Malaysia [57] found decreased antibiotic susceptibility of carriage strains. Because of the small sample size, we have interpreted these findings with caution. However, further assessment of the antibiotic susceptibility of meningococci is warranted because of the potential emergence of resistant strains that could complicate treatment, particularly in countries that do not have routine vaccination programs for primary prevention.

Few studies in our review assessed the effect of vaccination on carriage, which is also related to the limited number of countries that have incorporated meningococcal vaccines in national immunization programs (Table S1). Although meningococcal vaccination has led to a reduction in meningococcal disease [61], the effect of vaccination on carriage acquisition depends on the type of vaccine (i.e., polysaccharide vs. conjugate vaccines) [62]. In our review, a study from Salvador, Brazil, found a low rate of MenC carriage isolates in adolescents and young adults, which was attributed to a mass MenC vaccination campaign for 10 24 year olds [34]. Another study compared the phenotypes of isolates from healthy carriers in Cuba before and following widespread VAMENGOC-BC vaccination; this study found that MenB isolates predominated in the 
epidemic (i.e., pre-vaccination) period whereas nonserogroupable isolates became prominent in the post-vaccination period [47].

Interestingly, studies from Chile found a high rate of MenW cc11 among disease isolates, with only a very small percentage, if any, of carriage isolates attributed to cc11 $[40,41]$. The authors hypothesized that the low carriage of MenW cc11 in Chile could be attributed to enhanced virulence of this strain, allowing most infections to result in disease rather than carriage. This finding differs with those from studies from Burkina Faso [63] and Saudi Arabia [64], which found a positive correlation of MenW carriage prevalence and disease incidence. However, it is similar to those from studies of MenC in the United Kingdom, where carriage of cc11 was rare, but MenC was an important cause of disease [18], and studies of MenA in sub-Saharan Africa, where carriage was rare, but disease was common [65].

Risk factors associated with carriage were inconsistently assessed in studies from low and middle income countries of the Americas and Asia. In addition, few studies had adequate power to measure associations because of the low rates of carriage and the small sample sizes among subgroups. North American and European studies have found an association between smoking and carriage [14, 15], and a study from sub-Saharan Africa found an association between both passive and active smoking and carriage, the latter of which was statistically significant [12]. In comparison, our review found a significant association of carriage with passive smoking in only three studies from the Americas [33, 35, 42] and no significant association with active smoking.

A recently published systematic review and meta-analysis by Peterson and colleagues investigated serogroup-specific meningococcal carriage by age group [66]. Eligible carriage studies were carried out between 2007 and 2016 in healthy populations with defined age groups and geographical regions. A total of 65 studies were included in the review. Overall carriage rates from low and middle income countries of the Americas and Asia were generally similar to those identified in the current review. Of the 11 studies included from the Americas region, seven were set in low and middle income countries (Brazil, $n=4$; Chile, $n=2$; and Colombia, $n=1$ ); with the exception of a study carried out in Brazilian university students, which found a carriage rate of $71.1 \%$, the observed carriage in these studies ranged from $4.0 \%$ to $12.6 \%$. Almost all of the 32 studies from Asia were set in low and middle income countries (China, $n=29$; India, $n=1$; the Philippines, $n=1)$. Overall carriage rates observed in China varied from $0 \%$ to $33.7 \%$ and there was no discernable pattern with regards to year, province, or age group. The single studies from India and the Philippines reported rates of $1.5 \%$ and $1.6 \%$ to $9.1 \%$, respectively.

It is interesting to consider our findings in the context of meningococcal carriage data available from sub-Saharan Africa. In this region, carriage is highest in children, although patterns are not always consistent among studies [67]. In the systematic review by Peterson and colleagues, carriage in sub-Saharan Africa ranged from $1.7 \%$ in Burkina Faso (individuals aged 1-29 years) to $13.2 \%$ in the Gambia (individuals aged 10-18 years) [66]. In a metaanalysis of six of these African studies, the pattern of carriage across age groups was similar for all serogroups, with a peak consistently observed in 5-17 year olds. Consistent with these findings, a longitudinal study of sub-Saharan African countries found the overall carriage rate to be $2.4 \%$, with the highest rates in those 5-14 years of age [12]. This longitudinal study also found higher risk of meningococcal acquisition in those reporting a sore throat during the dry season, smoking tobacco (as described above), and exposure to wood smoke. In addition and consistent with our observations, a clear association between carriage prevalence and disease from these African data has not been identified [12, 67]. However, the introduction of the MenA conjugate vaccine MenAfriVac resulted in a substantial reduction in both disease and carriage acquisition [65].

The limitation of our review is the scope of the literature search, which was restricted to English-language articles within PubMed, thereby excluding some non-English language publications and articles on meningococcal carriage published in journals not indexed in 
PubMed. Further, any literature published locally by healthcare authorities or national surveillance programs (where available) was not captured by our search strategy. That said, it should be emphasized that the PubMed literature search likely included much of the data available on meningococcal carriage, particularly those from larger and contemporary studies. One particular limitation of this search strategy is that the current review includes two studies from China with only one including data outside of an outbreak setting [51, 53]. In contrast, the recently published systematic review of meningococcal carriage by Peterson and colleagues described above [66], which searched several databases (e.g., global health and Chinese literature databases), did not exclude any article based on language, and therefore included 29 studies from China (all of which were conducted between 2007 and 2016). However, compared with our literature review, this systematic review did not assess risk factors for carriage nor did it limit the analysis to low or middle income countries.

\section{CONCLUSIONS}

Future carriage studies in low and middle income countries of the Americas and Asia should incorporate larger representative populations, a wider age range, and additional countries in these regions. The countries included in our review are typically densely populated and have extensive socioeconomic, ethnic, and environmental diversity, suggesting that the epidemiology and risk factors for meningococcal carriage may also vary greatly between countries. Thus, the lack of contemporary and comprehensive carriage data in low and middle income countries of the Americas and Asia is a major unmet need. Such data, in combination with high quality disease surveillance, are needed to better elucidate the at-risk populations for disease and transmission so that meningococcal vaccine strategies that directly prevent disease and maximize herd protection can be developed based on the specific needs and characteristics of a particular country.

\section{ACKNOWLEDGEMENTS}

Funding. This work and the Rapid Service Fees were sponsored by Pfizer Inc.

Authorship. All named authors meet the International Committee of Medical Journal Editors (ICMJE) criteria for authorship for this article, take responsibility for the integrity of the work as a whole, and have given their approval for this version to be published.

Medical Writing and Editorial Assistance. Editorial and medical writing assistance in the preparation of this article was provided by Tricia Newell, PhD, of Complete Healthcare Communications, LLC (North Wales, PA), a CHC Group Company. Support for this assistance was funded by Pfizer Inc.

Disclosures. Lidia Serra and Jessica Presa are employees of Pfizer Inc and may hold stock or stock options. Hannah Christensen has received support from Sanofi-Pasteur and consultancy fees from IMS Health and AstraZeneca all paid to her employer. She is supported by the NIHR Health Protection Research Unit in Evaluation of Interventions at the University of Bristol. Caroline Trotter has received consulting fees from GSK in 2018, outside of the submitted work. The views expressed in this article are those of the author(s) and not necessarily those of the NHS, the NIHR, or the Department of Health and Social Care.

Compliance with Ethics Guidelines. This article is based on previously conducted studies and does not contain any studies with human participants or animals performed by any of the authors.

Data Availability. Data sharing is not applicable to this article as no datasets were generated or analyzed during the current study.

Open Access. This article is licensed under a Creative Commons Attribution-NonCommercial 4.0 International License, which permits any non-commercial use, sharing, adaptation, 
distribution and reproduction in any medium or format, as long as you give appropriate credit to the original author(s) and the source, provide a link to the Creative Commons licence, and indicate if changes were made. The images or other third party material in this article are included in the article's Creative Commons licence, unless indicated otherwise in a credit line to the material. If material is not included in the article's Creative Commons licence and your intended use is not permitted by statutory regulation or exceeds the permitted use, you will need to obtain permission directly from the copyright holder. To view a copy of this licence, visit http://creativecommons.org/licenses/by$\mathrm{nc} / 4.0 /$.

\section{REFERENCES}

1. Stephens DS, Greenwood B, Brandtzaeg P. Epidemic meningitis, meningococcaemia, and Neisseria meningitidis. Lancet. 2007;369:2196-210.

2. Collaborators GBDM. Global, regional, and national burden of meningitis, 1990-2016: a systematic analysis for the Global Burden of Disease Study 2016. Lancet Neurol. 2018;17:1061-82.

3. Institute for Health Metrics and Evaluation. GHDx. University of Washington. http://ghdx.healthdata. org/gbd-results-tool. Accessed 10 Sep 2018.

4. Thompson MJ, Ninis N, Perera R, et al. Clinical recognition of meningococcal disease in children and adolescents. Lancet. 2006;367:397-403.

5. Pace D, Pollard AJ. Meningococcal disease: clinical presentation and sequelae. Vaccine. 2012;30:B3-9.

6. Jafri RZ, Ali A, Messonnier NE, et al. Global epidemiology of invasive meningococcal disease. Popul Health Metr. 2013;11:11-7.

7. National Center for Immunization and Respiratory Diseases. Enhanced meningococcal disease surveillance report, 2017 (CS283195). Atlanta: Centers for Disease Control and Prevention; 2017.

8. Institute of Environmental Science and Research Ltd. 2018 invasive meningococcal disease report. https://surv.esr.cri.nz/surveillance/Meningococcal_ disease.php?we_objectID=4876. 2019.

9. Booy R, Gentile A, Nissen M, Whelan J, Abitbol V. Recent changes in the epidemiology of Neisseria meningitidis serogroup $\mathrm{W}$ across the world, current vaccination policy choices and possible future strategies. Hum Vaccin Immunother. 2019;15: $470-80$

10. Kimberlin DW, Brady MT, Jackson MA, Long SS, editors. Red book 2015. 30th ed. Elk Grove Village: American Academy of Pediatrics; 2015.

11. Ceyhan M, Celik M, Demir ET, et al. Acquisition of meningococcal serogroup $\mathrm{W}-135$ carriage in Turkish Hajj pilgrims who had received the quadrivalent meningococcal polysaccharide vaccine. Clin Vaccine Immunol. 2013;20:66-8.

12. Cooper LV, Robson A, Trotter CL, et al. Risk factors for acquisition of meningococcal carriage in the African meningitis belt. Trop Med Int Health. 2019;24:392-400.

13. Christensen $\mathrm{H}$, May $\mathrm{M}$, Bowen L, Hickman $\mathrm{M}$, Trotter CL. Meningococcal carriage by age: a systematic review and meta-analysis. Lancet Infect Dis. 2010;10:853-61.

14. Bruce MG, Rosenstein NE, Capparella JM, et al. Risk factors for meningococcal disease in college students. JAMA. 2001;286:688-93.

15. MacLennan J, Kafatos G, Neal K, et al. Social behavior and meningococcal carriage in British teenagers. Emerg Infect Dis. 2006;12:950-7.

16. Swain CL, Martin DR. Survival of meningococci outside of the host: implications for acquisition. Epidemiol Infect. 2007;135:315-20.

17. Deasy AM, Guccione E, Dale AP, et al. Nasal inoculation of the commensal Neisseria lactamica inhibits carriage of Neisseria meningitidis by young adults: a controlled human infection study. Clin Infect Dis. 2015;60:1512-20.

18. Maiden MC, Ibarz-Pavon $\mathrm{AB}$, Urwin $\mathrm{R}$, et al. Impact of meningococcal serogroup $\mathrm{C}$ conjugate vaccines on carriage and herd immunity. J Infect Dis. 2008;197:737-43.

19. Borrow R, Alarcon P, Carlos J, et al. The global meningococcal initiative: global epidemiology, the impact of vaccines on meningococcal disease and the importance of herd protection. Expert Rev Vaccines. 2017;16:313-28.

20. International Monetary Fund. Fiscal monitor: how to mitigate climate change. Washington: International Monetary Fund; 2019.

21. Safadi MA, O'Ryan M, Valenzuela Bravo MT, et al. The current situation of meningococcal disease in Latin America and updated global meningococcal 
initiative (GMI) recommendations. Vaccine. 2015;33:6529-36.

22. Ruttimann RW, Gentile A, Parra MM, et al. A consensus statement: meningococcal disease among infants, children and adolescents in Latin America. Pediatr Infect Dis J. 2014;33:284-90.

23. Safadi MA, Gonzalez-Ayala S, Jakel A, et al. The epidemiology of meningococcal disease in Latin America 1945-2010: an unpredictable and changing landscape. Epidemiol Infect. 2013;141:447-58.

24. World Health Organization. WHO vaccine-preventable diseases: monitoring system. 2018 global summary. http://apps.who.int/immunization monitoring/globalsummary/schedules. Accessed 12 Sep 2018.

25. Moraes C, Moraes JC, Silva GD, Duarte EC. Evaluation of the impact of serogroup $\mathrm{C}$ meningococcal disease vaccination program in Brazil and its regions: a population-based study, 2001-2013. Mem Inst Oswaldo Cruz. 2017;112:237-46.

26. Safadi MA, Berezin EN, Oselka GW. A critical appraisal of the recommendations for the use of meningococcal conjugate vaccines. J Pediatr (Rio J). 2012;88:195-202.

27. Cardoso CW, Pinto LL, Reis MG, Flannery B, Reis JN. Impact of vaccination during an epidemic of serogroup C meningococcal disease in Salvador, Brazil. Vaccine. 2012;30:5541-6.

28. Olea A, Matute I, Gonzalez C, et al. Case-control study of risk factors for meningococcal disease in Chile. Emerg Infect Dis. 2017;23:1070-8.

29. Valenzuela MT, Mañalich J, Díaz J, et al. Plan de acción nacional frente a la emergencia de la cepa W-135 responsable de enfermedad meningocócica invasora en Chile, 2012-2013. Rev Med Chile. 2019;147:776-86.

30. Ministry of Health of Chile. Consideraciones del CAVEI para la promoción de al adeherencia a la vacunación. https://vacunas.minsal.cl/wp-content/ uploads/2018/05/CAVEI-Adherencia_24Mayo2018. pdf. Accessed 16 Aug 2019.

31. Ministry of Health of Chile. Calendario de Vacunación 2014. https://vacunas.minsal.cl/ informacion-a-la-comunidad/calendario-devacunacion/calendario-de-vacunacion-2014/. Accessed 16 Aug 2019.

32. Climent Y, Yero D, Martinez I, et al. Clonal distribution of disease-associated and healthy carrier isolates of Neisseria meningitidis between 1983 and 2005 in Cuba. J Clin Microbiol. 2010;48:802-10.
33. Nunes AM, Ribeiro GS, Ferreira IE, et al. Meningococcal carriage among adolescents after mass meningococcal $\mathrm{C}$ conjugate vaccination campaigns in Salvador, Brazil. PLoS One. 2016;11:e0166475.

34. Moura A, Kretz CB, Ferreira IE, et al. Molecular characterization of Neisseria meningitidis isolates recovered from 11-19-year-old meningococcal carriers in Salvador, Brazil. PLoS One. 2017;12: e0185038.

35. Cassio de Moraes J, Kemp B, de Lemos AP, et al. Prevalence, risk factors and molecular characteristics of meningococcal carriage among Brazilian adolescents. Pediatr Infect Dis J. 2015;34:1197-202.

36. Weckx LY, Puccini RF, Machado A, et al. A crosssectional study assessing the pharyngeal carriage of Neisseria meningitidis in subjects aged 1-24 years in the city of Embu das Artes, Sao Paulo, Brazil. Braz J Infect Dis. 2017;21:587-95.

37. Araya P, Fernandez J, Del Canto F, et al. Neisseria meningitidis ST-11 clonal complex, Chile 2012. Emerg Infect Dis. 2015;21:339-41.

38. Safadi MA, Carvalhanas TR, Paula de Lemos A, et al. Carriage rate and effects of vaccination after outbreaks of serogroup C meningococcal disease, Brazil, 2010. Emerg Infect Dis. 2014;20:806-11.

39. Barroso D. Neisseria meningitidis nasopharynx colonization of diseased patients on presentation and on discharge. Trop Doct. 1999;29:108-9.

40. Rubilar PS, Barra GN, Gabastou JM, et al. Increase of Neisseria meningitidis $\mathrm{W}: \mathrm{cc} 11$ invasive disease in Chile has no correlation with carriage in adolescents. PLoS ONE. 2018;13:e0193572.

41. Rodriguez P, Alvarez I, Torres MT, et al. Meningococcal carriage prevalence in university students, 1824 years of age in Santiago, Chile. Vaccine. 2014;32:5677-80.

42. Diaz J, Carcamo M, Seoane M, et al. Prevalence of meningococcal carriage in children and adolescents aged 10-19 years in Chile in 2013. J Infect Public Health. 2016;9:506-15.

43. Moreno J, Hidalgo M, Duarte C, et al. Characterization of carriage isolates of Neisseria meningitides in the adolescents and young adults population of Bogota (Colombia). PLoS ONE. 2015;10:e0135497.

44. Espinosa de los Monteros LE, Aguilar-Ituarte F, Jimenez-Rojas LV, et al. Prevalence of Neisseria meningitidis carriers in children under five years of age and teenagers in certain populations of Mexico City. Salud Publica Mex. 2009;51:114-8. 
45. Chamorro G, Ibarz-Pavon AB, Kawabata A, et al. Carriage of Neisseria meningitidis and other Neisseria species among children and young adults in Paraguay. J Med Microbiol. 2019.

46. Martinez Mota I, Gonzales GS, Gutierrez NN, et al. Caracterización de cepas de Neisseria meningitidis aisladas de portadores en Cuba durante 20 años. Rev Cubana Med Trop. 2006;58:124-33.

47. Martinez Motas I, Sierra Gonzalez G, Nunez Gutierrez N, et al. Characterization of Neisseria meningitidis strains isolated from carriers in Cuba during 20 years. Rev Cubana Med Trop. 2006;58: 124-33.

48. Gonzales ML, Bianco V, Vyse A. Meningococcal carriage in children and young adults in the Philippines: a single group, cross-sectional study. Epidemiol Infect. 2017;145:126-32.

49. Borrow R, Lee JS, Vazquez JA, et al. Meningococcal disease in the Asia-Pacific region: findings and recommendations from the global meningococcal initiative. Vaccine. 2016;34:5855-62.

50. Chiou CS, Liao JC, Liao TL, et al. Molecular epidemiology and emergence of worldwide epidemic clones of Neisseria meningitidis in Taiwan. BMC Infect Dis. 2006;6:25.

51. Zhou H, Gao Y, Xu L, et al. Distribution of serogroups and sequence types in disease-associated and carrier strains of Neisseria meningitidis isolated in China between 2003 and 2008. Epidemiol Infect. 2012;140:1296-303.

52. Sinclair D, Preziosi MP, Jacob John T, Greenwood B. The epidemiology of meningococcal disease in India. Trop Med Int Health. 2010;15:1421-35.

53. Zhang J, Zhou HJ, Xu L, et al. Molecular characteristics of Neisseria meningitidis isolated during an outbreak in a jail: association with the spread and distribution of ST-4821 complex serogroup C clone in China. Biomed Environ Sci. 2013;26:331-7.

54. Jha D, Ghosh MK. Epidemiology of meningococcal carrier state amongst recruits of a military training centre. J Commun Dis. 1995;27:250-5.

55. Ichhpujani RL, Mohan R, Grover SS, Joshi PR, Kumari S. Nasopharyngeal carriage of Neisseria meningitidis in general population and meningococcal disease. J Commun Dis. 1990;22:264-8.

56. Paul VK, Bhujwala RA, Ghai OP, et al. Nasopharyngeal carriers among contact of patients of
Neisseria meningitidis infection in Delhi. Indian J Med Res. 1987;86:429-32.

57. Rohani MY, Ahmad Afkhar F, Amir MA, et al. Serogroups and antibiotic susceptibility patterns of Neisseria meningitidis isolated from army recruits in a training camp. Malays J Pathol. 2007;29:91-4.

58. Thapa S, Gokhale S, Sharma AL, et al. Burden of bacterial upper respiratory tract pathogens in school children of Nepal. BMJ Open Respir Res. 2017;4:e000203.

59. Danchaivijitr S, Leelarasmee A, Sankaburanuraksa S, et al. Meningococcal carriers in school children. J Med Assoc Thai. 1988;71:537-40.

60. Coch Gioia CA, Silva de Lemos AP, Outeiro Gorla $\mathrm{MC}$, et al. Detection of Neisseria meningitidis in asymptomatic carriers in a university hospital from Brazil. Rev Argent Microbiol. 2015;47:322-7.

61. Pelton SI. The global evolution of meningococcal epidemiology following the introduction of meningococcal vaccines. J Adolesc Health. 2016;59: S3-11.

62. Borrow R, Caugant DA, Ceyhan $\mathrm{M}$, et al. Meningococcal disease in the Middle East and Africa: findings and updates from the global meningococcal initiative. J Infect. 2017;75:1-11.

63. Kristiansen PA, Ba AK, Sanou I, et al. Phenotypic and genotypic characterization of meningococcal carriage and disease isolates in Burkina Faso after mass vaccination with a serogroup a conjugate vaccine. BMC Infect Dis. 2013;13:363.

64. Balkhy HH, Memish ZA, Osoba AO. Meningococcal carriage among local inhabitants during the pilgrimage 2000-2001. Int J Antimicrob Agents. 2003;21:107-11.

65. Daugla DM, Gami JP, Gamougam K, et al. Effect of a serogroup A meningococcal conjugate vaccine (PsA-TT) on serogroup A meningococcal meningitis and carriage in Chad: a community study (corrected). Lancet. 2014;383:40-7.

66. Peterson ME, Li Y, Bita A, et al. Meningococcal serogroups and surveillance: a systematic review and survey. J Glob Health. 2019;9:010409.

67. Trotter CL, Greenwood BM. Meningococcal carriage in the African meningitis belt. Lancet Infect Dis. $2007 ; 7: 797-803$. 